\title{
Structures, processes and outcomes of specialist critical care nurse education: an integrative review
}

\section{Abstract}

Objectives: To review and synthesise international literature to reveal the contemporary structures, processes and outcomes of critical care nurse (CCN) education.

Method: An integrative review on specialist critical care education was guided by Whittemore and Knafl's integrative review steps: problem-identification; literature search; and data evaluation, analysis and presentation. Donabedian's Quality Framework (Structure-Process-Outcome) provided a useful analytical lens and structure for the reporting of findings.

Results: 1) Structures for CCN education incorporated transition-to-practice and ongoing education programs typically offered by hospitals and health services, and university level graduate certificate, diploma and masters programs. Structural expectations included a standard core curriculum, clinically credible academic staff and courses compliant with a higher education framework. Published workforce standards and policies were important structures for the practice learning environment. 2) Processes included incremental exposure to increasing patient acuity; consistent and appropriately supported and competent hospital-based preceptors/assessors; courses delivered with a flexible, modular approach; curricula that support non-technical skills and patient and family-centred care; stakeholder engagement between the education provider and the clinical setting to guide course planning, evaluation and revalidation; and evidence-based measurement of clinical capabilities/competence. 3) Outcomes included articulation of the scope and levels of graduate attributes and professional activities associated with each level. The role of higher degree research programs for knowledge-creation and critical care academic leadership was noted.

Conclusions: Provision of high quality critical care education is multifaceted and complex. These findings provide information for healthcare organisations and education providers. This may enable 
best practice structures and processes for critical care specialist training that meets the needs of industry and safely supports developing CCN expertise. There is an acknowledged tension between the expectations of governing bodies for policies, standards and position statements to enhance quality and reduce care variance, and the availability of high-quality evidence to underpin these across international contexts.

Keywords: critical care nurses; education; integrative review; intensive care nurses; literature review; ongoing education; postgraduate education; specialty practice; transition to practice programs. 


\section{Introduction}

Specialist critical care nurses (CCN) need the capability to integrate advanced theoretical knowledge and practical and interpersonal skills to meet critically ill patients' care needs. Critically ill patients often present with multiple organ dysfunction, haemodynamic instability, complex pharmacological regimens and both patients and families are vulnerable to psychological distress. ${ }^{1,2}$ Effective care provided by CCNs requires not only direct and comprehensive clinical care, but also a collaborative approach to communication and problem solving to enhance the combined critical thinking potential within a supportive interdisciplinary team. ${ }^{3}$

Some critical care professional organisations argue that the complexity of the $\mathrm{CCN}$ role requires a structured and continuous program of education from entry-level practice, through to postgraduate courses (i.e. delivered by a university or other accredited higher education provider). ${ }^{4,5}$ In this review, postgraduate qualifications are defined as a minimum of Australian Qualifications Framework (AQF) level 8 (postgraduate certificate or postgraduate diploma). ${ }^{6}$ This formal education aims to provide the public with confidence in the CCN's capacity and capability for safe high quality care. ${ }^{5}$ The evidence for the impact of postgraduate education is mixed. For example, a recent systematic review reported nurses holding a Master level qualification within their clinical speciality contributed to fewer emergency, intensive care unit (ICU) and hospital readmissions and improved both survival outcomes and mental health outcomes. ${ }^{7}$ Countering these conclusions are criticisms of past literature on postgraduate education outcomes ${ }^{8,9}$ asserting methodological limitations and a scarcity of causal links and patient outcome indicators. ${ }^{10}$ There is stronger evidence for benefits to individual nurses and health services with postgraduate education credited with developing a higher level of critical thinking, increased confidence as an interdisciplinary team member and personal and professional growth. ${ }^{10}$ The potential role of postgraduate education as a driver of improved registered nurse retention has been noted in national workforce documents ${ }^{11,10}$ and discussion papers. ${ }^{12,13}$ 
As critical care practice and technologies are dynamic, formal CCN qualifications should be augmented with continuing education to achieve and maintain an advanced or specialist level of evidence-based CCN practice. While outcomes of continuing education are difficult to measure, ${ }^{14}$ and studies vary in quality, a review from the United States (US) on the continuing education of health professionals provided some evidence of improved knowledge and skill base, a capacity to change attitudes and behaviours, and subsequent improvement in clinical outcomes. ${ }^{15}$

The impetus for this current review was to inform a revision of the Australian College of Critical Care Nurses' (ACCCN) Position Statement on the Provision of Critical Care Nurse Education. ${ }^{16}$ This integrative review builds on a 2012 literature review ${ }^{17}$ that examined the qualifications of CCN staff, and postgraduate education and standards across Australia, the United States (US), Canada, the United Kingdom (UK) and New Zealand (NZ). While the diversity and lack of comparable data made it difficult to quantify differences internationally, the importance of optimising the number of staff with post-registration qualifications and of minimum practice standards to guide content for CCN qualifications were elements of consensus. This work informed the subsequent development of minimum practice standards for the Australian CCN qualification and defined CCN education graduate practice outcomes. ${ }^{18,19}$ The robustness of the empirical mixed-methods approach used to develop the Australian Practice Standards from the mid-1990s, in comparison to the expert panel method used in the US, Canada, UK and NZ was a key finding. ${ }^{17}$

This Australian focus on educational relevance, quality and standards reflects a desire internationally to define minimum practice standards for $\mathrm{CCN}$ postgraduate course graduates, ${ }^{5,20}$ to articulate a clinical practice standard as a course outcome, ${ }^{18,19,21,22}$ to establish expectations between industry and course providers ${ }^{23,24}$ and to propose minimum criteria for a CCN qualification. ${ }^{4,25}$ Globally, postgraduate qualifications frameworks further define education levels to allow for comparability and transferability of qualifications, and to outline the necessary credentials and further continuing education of assessors and trainers. ${ }^{6,26-30}$ 
Given the implications for patient safety, ${ }^{31,32}$ the governance expectations of government, health services and health departments ${ }^{33}$ and the need to develop and retain a specialist CCN workforce, ${ }^{34,}$ 35 the aim of this review of international literature was to reveal the contemporary structures, processes and outcomes of specialist critical care education.

\section{Material and methods}

\section{The theoretical framework}

This integrative review was guided by Donabedian's ${ }^{36}$ Quality Framework (Structure-Process-Outcome (SPO) for health services evaluation. While other quality frameworks for vocational adult education exist, ${ }^{37,38}$ the specific needs of the health industry seem best served by a model that is designed to encompass the complexities and concerns of the health policy environment, while maintaining patient safety and comfort as a central goal. The utility of using such a structure is not simply to provide a theoretical lens, but is based on the premise that improvements in underlying structures can lead to demonstrated advancements in process, and subsequently, in measurable improvements in outcomes. These links have been demonstrated in clinical evaluation of health programs. ${ }^{39,40}$ More recently, the potential for Donabedian's approach for use as a quality framework underpinning design and evaluation of health professional education has been proposed. ${ }^{41,42}$ Donabedian's framing was applied as a way of structuring our report rather than as a filter for inclusion/exclusion of studies or findings, with all available contemporary grey literature and research reports considered.

The SPO framework provides a flexible outline that incorporates three inter-related concepts that we applied to an education context: 1) Structures of education acknowledge important organisational elements such as guiding standards, national qualifications frameworks, physical facilities, personnel, available equipment, technology and financial/governance processes; 2) Processes of education include curriculum content and strategies for education delivery and competence assessment 
(inclusive of transition to practice, postgraduate and ongoing education processes). These are reliant on the aforementioned structures for necessary resources and mechanisms; and 3) Outcomes include enhanced graduate scope and attributes that should, in turn, promote improved service outcomes, and ultimately, enhance patient and family outcomes. ${ }^{43}$

\section{Methods}

The following research questions guided our review:

1. What are the contemporary structures (such as courses, programs and policy/standards documents) for CCN specialist education internationally?

2. What are the facilitating processes for $\mathrm{CCN}$ specialist education? (How are these courses and programs delivered?)

3. What are the outcomes of specialist CCN education internationally?

Whittemore and Knafl's integrative review methodology ${ }^{44}$ enabled a synthesis of findings from documents with diverse methodologies and perspectives. This wide-ranging sampling frame and the multiple purposes of included sources, (in our case, research papers, a reflective analysis, standards and consensus statements) comprehensively informed the complexity of concepts, theories and problems of relevance to contemporary $\mathrm{CCN}$ education.

The stages and activities of this review included problem identification, literature search, data evaluation, data analysis, and presentation. ${ }^{44}$ One author (LW) developed the search strategy (see Figure 1) and conducted the initial search according to agreed inclusion/exclusion criteria. A second author (JG) confirmed the search steps and publication inclusion/exclusion. 
Figure 1: PRISMA FLOW DIAGRAM ${ }^{45}$
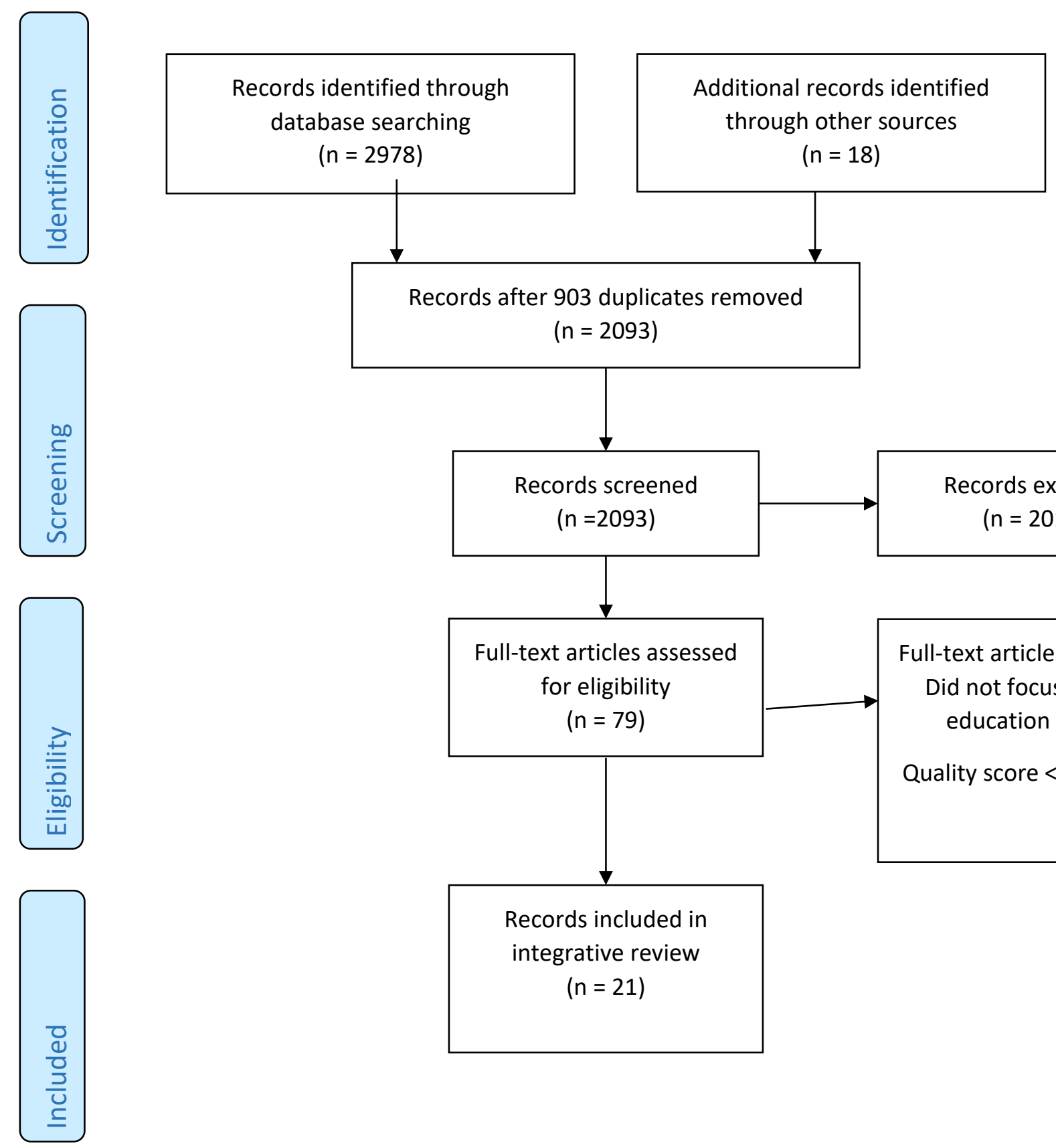

Records after 903 duplicates removed

$(n=2093)$

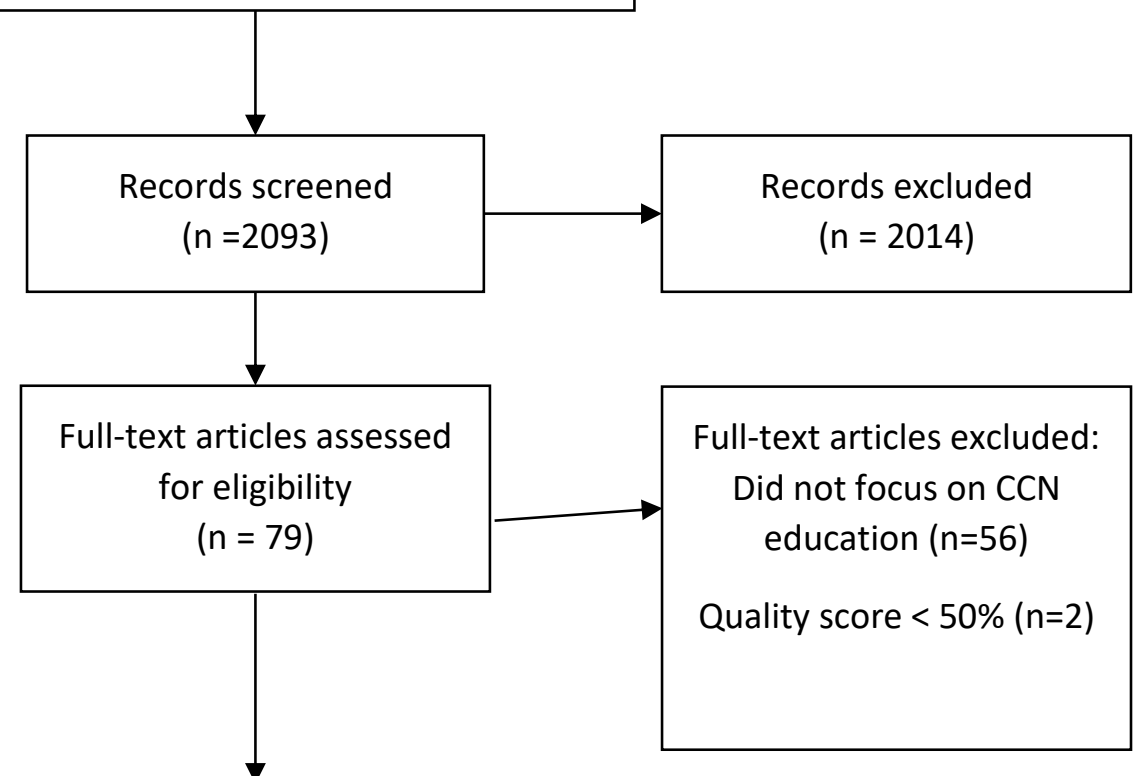

Records included in integrative review $(n=21)$ 
The Cumulative Index to Nursing and Allied Health Literature (CINAHL) Plus, Ovid Nursing, Medline, Embase, Joanna Briggs Institute, and Google Scholar databases were searched for articles on critical care nursing and education. A Boolean strategy was applied with database-specific variations on the search terms 'critical care nursing' OR 'intensive care nursing' AND 'graduate education' OR 'postgraduate education' $O R$ 'education' $O R$ 'standards' $O R$ 'trends'. Included manuscripts were published in English and had a focus on education programs to enhance the capability of registered nurses working in critical care environments. Publications were excluded if they did not meet an agreed quality score.

As a comprehensive literature review was published in $2012,{ }^{17}$ our search covered the period between January 2013 and December 2016. This resulted in identification of 2093 records after removal of 903 duplicates. After screening of abstracts, seventy-nine articles were assessed for eligibility. Fifty-six did not explore $\mathrm{CCN}$ education in methods or results and were removed.

The quality appraisal tools were agreed upon and authors worked in pairs to conduct the initial quality appraisal, with a final verification by the first author. Quality appraisal of publications with diverse methodologies required a range of critical appraisal checklists: the Mixed Methods Appraisal Tool $(\text { MMAT })^{46}$ for empirical research papers, the Narrative Review Checklist ${ }^{47}$ for narrative review papers and the JBI (Joanna Briggs Institute) Checklist for Text and Opinion ${ }^{48}$ for professional standards and position statements. Given the diversity of papers, we took a pragmatic approach to the issue of quality: quality scores were converted to a percentage and publications were excluded $(n=2)$ where the quality score was less than $50 \%$. The characteristics and quality appraisal details of included empirical studies and grey literature are documented in Tables 1 and 2 respectively.

The analysis of data was a two-phase process. Data were firstly organised deductively using Donabedian's Structure-Process-Outcome (SPO) mode $^{36}$ as an analytical lens. Data were then themed inductively within these categories. Findings were corroborated and agreed amongst authors. Papers that created uncertainty were discussed among the research team until consensus was reached. 
Table 1. Characteristics of empirical studies (supplementary file)

\begin{tabular}{|c|c|c|c|c|c|c|c|}
\hline Authors & $\begin{array}{l}\text { Study } \\
\text { design }\end{array}$ & Purpose & Setting & Sample & Methods & $\begin{array}{l}\text { Quality } \\
\text { assessment } \\
\text { tool used \& } \\
\text { quality score }\end{array}$ & Findings \\
\hline $\begin{array}{l}\text { Adams, } \\
\text { Alexander, } \\
\text { Chisari, et } \\
\text { al. } 2015^{49}\end{array}$ & $\begin{array}{l}\text { Descriptive } \\
\text { qualitative } \\
\text { study }\end{array}$ & $\begin{array}{l}\text { To assess } \\
\text { effectiveness of a } \\
\text { 6-month CCN } \\
\text { residency (TTP) } \\
\text { program }\end{array}$ & $\begin{array}{l}\text { Magnet }^{\circledR} \\
\text { Hospital in } \\
\text { Boston, USA }\end{array}$ & $\begin{array}{l}\text { Criterion sample } \\
\text { of } 34 \text { new } \\
\text { graduate nurses, } \\
\text { five nurse } \\
\text { managers \& five } \\
\text { clinical nurse } \\
\text { specialists }\end{array}$ & $\begin{array}{l}12 \text { focus group } \\
\text { interviews audio- } \\
\text { recorded, only } 8 \\
\text { transcribed \& fully } \\
\text { analysed (due to } \\
\text { reaching data } \\
\text { saturation) \& } \\
\text { remaining } 4 \text { listened } \\
\text { to, to confirm } \\
\text { themes. Qualitative } \\
\text { content analysis. }\end{array}$ & $\begin{array}{l}\text { MMAT 75\% } \\
\text { Researcher } \\
\text { role/relationship } \\
\text { to participants } \\
\text { not described. }\end{array}$ & $\begin{array}{l}\text { Structure: Core clinical content of the TTP Program was complemented } \\
\text { by content on professional practice issues. } \\
\text { Process: There was an opportunity to extend duration of a student's } \\
\text { program where necessary. Difficult to schedule nurses with preceptors; } \\
\text { lack of continuity of preceptors. A need for clear role \& responsibility } \\
\text { delineation to enhance program continuity. Needs processes for } \\
\text { stakeholder feedback. Outcome: New graduates in program blocked local } \\
\text { opportunities for new recruitment \& projects. Nurses were proud of their } \\
\text { progress \& appreciated ongoing access to experts after program. Nurses } \\
\text { were stressed by uncertainties of future work environment, personal } \\
\text { capabilities \& future roles. }\end{array}$ \\
\hline $\begin{array}{l}\text { Baid \& } \\
\text { Hargreaves, } \\
2015^{32}\end{array}$ & $\begin{array}{l}\text { Reflective } \\
\text { analysis }\end{array}$ & $\begin{array}{l}\text { To reflect on how } \\
\text { one postgraduate } \\
\text { critical care } \\
\text { nursing course } \\
\text { enables safe, high } \\
\text { quality care for } \\
\text { patients \& families }\end{array}$ & $\begin{array}{l}\text { University in } \\
\text { the United } \\
\text { Kingdom }\end{array}$ & $\mathrm{N} / \mathrm{A}$ & $\begin{array}{l}\text { Reflection guided \& } \\
\text { structured according } \\
\text { to Rolfe's framework } \\
\text { for reflective } \\
\text { practice. }\end{array}$ & $\begin{array}{l}\text { JBI Checklist } \\
\text { for text \& } \\
\text { opinion } 7 / 7 \\
(100 \%)\end{array}$ & $\begin{array}{l}\text { Structure: Quality Assurance Standards can guide \& optimise clinical skill } \\
\text { assessment. Critical care quality indicators should inform postgraduate } \\
\text { programs. Process: Informed practice draws on published documents } \\
\text { (e.g. research, code of ethics, guidelines \& care bundles), previous } \\
\text { experience (TTP program \& minimum 1-year ICU experience before } \\
\text { postgraduate enrolment) \& patient \& family stories (published, videoed or } \\
\text { guest speakers). Assessment tasks should develop informed practice: } \\
\text { audit skills, workplace \& conference presentations, \& publications. } \\
\text { Simulation useful for formative assessment, non-technical skills \& for multi- } \\
\text { disciplinary learning. Outcome: Simulation is reliant on } \\
\text { fidelity/validity/reliability. Non-technical skills address human factors \& } \\
\text { develop cognitive \& interpersonal skills. }\end{array}$ \\
\hline $\begin{array}{l}\text { Bortolotto, } \\
2015^{50}\end{array}$ & $\begin{array}{l}\text { Mixed } \\
\text { methods } \\
\text { evaluation } \\
\text { study }\end{array}$ & $\begin{array}{l}\text { To describe \& } \\
\text { evaluate a critical } \\
\text { care TTP program } \\
\text { (clinical orientation } \\
\text { program for new } \\
\text { graduate nurses) }\end{array}$ & $\begin{array}{l}\text { Single } \\
\text { intensive care } \\
\text { unit in the } \\
\text { USA }\end{array}$ & $\begin{array}{l}175 \text { new } \\
\text { graduate nurses } \\
\text { who began a } \\
\text { transition to } \\
\text { critical care } \\
\text { practice program } \\
\text { between 2008- } \\
2013 \text {. }\end{array}$ & $\begin{array}{l}\text { Course completion } \\
\text { rates, New Graduate } \\
\text { retention rates, falls } \\
\text { \& infection rates, } \\
\text { patient satisfaction } \\
\text { scores, pain } \\
\text { management } \\
\text { outcomes. }\end{array}$ & MMAT $100 \%$ & $\begin{array}{l}\text { Structure: 12-month hospital-wide new graduate program including a 6- } \\
\text { month redesigned / formalised critical care TTP program. Each new } \\
\text { graduate assigned a small team of consistent assessors (covering shifts). } \\
\text { Process: Didactic study; standardised modules from American } \\
\text { Association of Critical Care Nurses; integration of case studies; } \\
\text { incremental staging of complexity of clinical exposure (facilitated by a } \\
\text { staging checklist); active development of preceptors \& student feedback } \\
\text { training; simulated clinical scenarios; facilitated group discussions. } \\
\text { Outcome: High course completion rates ( } 94 \% \text { ). Two-year retention rate } \\
\text { increased from } 62 \% \text { to }>95 \% \text {. Inferred costs savings. Patient sensitive } \\
\text { outcomes indicated program success. }\end{array}$ \\
\hline
\end{tabular}




\begin{tabular}{|c|c|c|c|c|c|c|c|}
\hline $\begin{array}{l}\text { Bromley, } \\
201451\end{array}$ & $\begin{array}{l}\text { Narrative } \\
\text { literature } \\
\text { review }\end{array}$ & $\begin{array}{l}\text { To understand the } \\
\text { concept of } \\
\text { competence \& its } \\
\text { evaluation for } \\
\text { students of } \\
\text { postgraduate } \\
\text { neonatal critical } \\
\text { care courses }\end{array}$ & $\begin{array}{l}\text { Neonatal } \\
\text { intensive care } \\
\text { programs } \\
\text { internationally }\end{array}$ & $\mathrm{N} / \mathrm{A}$ & $\begin{array}{l}\text { Considered the } \\
\text { terms competence, } \\
\text { competent, } \\
\text { competency \& } \\
\text { competencies. } \\
\text { Compared } \\
\text { perspectives of } \\
\text { competence } \\
\text { between clinicians, } \\
\text { patients \& families. } \\
\text { Critiqued direct } \\
\text { observation, self - } \\
\text { assessment \& } \\
\text { practice portfolios as } \\
\text { measures of } \\
\text { competence. }\end{array}$ & $\begin{array}{l}\text { Elsevier } \\
\text { Narrative } \\
\text { review checklist } \\
5 / 7(71 \%) \\
\text { Did not identify } \\
\text { as a narrative } \\
\text { review in the } \\
\text { title. The } \\
\text { process for } \\
\text { identifying } \\
\text { included } \\
\text { studies not } \\
\text { specified. }\end{array}$ & $\begin{array}{l}\text { Structure: Nursing \& Midwifery Board of Australia Competency Standards } \\
\text { for the Registered Nurse; Australian Standards for Neonatal Nurses; No } \\
\text { agreement on expected postgraduate attributes of neonatal course } \\
\text { students; Many competence evaluation tools are unreliable \& non- } \\
\text { validated. Process: Direct observation; self-assessment; practice } \\
\text { portfolios; competency assessment tools; Use of competency standards as } \\
\text { a measure of competence. Outcomes: Competence is complex \& } \\
\text { multidimensional \& incorporates cognitive, affective \& psychomotor } \\
\text { aspects. Direct observation has poor inter-rater reliability; in self- } \\
\text { assessment, incompetent people tend to overrate abilities \& competent } \\
\text { people tend to underrate their skills. Competency standards not developed } \\
\text { or validated as measures of competence and may not be useful for learner } \\
\text { level competence. Patients prioritise interpersonal aspects of neonatal } \\
\text { care \& 'feeling cared for'. }\end{array}$ \\
\hline $\begin{array}{l}\text { Currey, } \\
\text { Eustace, } \\
\text { Oldland, et } \\
\text { al, } 2015^{52} \text {. }\end{array}$ & $\begin{array}{l}\text { Mixed } \\
\text { methods } \\
\text { study }\end{array}$ & $\begin{array}{l}\text { To describe } \\
\text { experiences \& } \\
\text { perceptions of } \\
\text { team-based } \\
\text { learning }\end{array}$ & $\begin{array}{l}\text { One class } \\
\text { from one } \\
\text { Australian } \\
\text { University. }\end{array}$ & $\begin{array}{l}32 \text { postgraduate } \\
\text { critical care } \\
\text { students }\end{array}$ & $\begin{array}{l}\text { Extended response } \\
\text { questionnaire \& field } \\
\text { observations. } \\
\text { Analysis using the } \\
\text { constant comparison } \\
\text { technique. Thematic } \\
\text { analysis }\end{array}$ & $\begin{array}{l}\text { MMAT 75\% } \\
\text { (non-validated } \\
\text { survey tool). }\end{array}$ & $\begin{array}{l}\text { Structure: Team-based learning approach. Teacher controls content \& } \\
\text { guides self-managing teams in active learning process. Process: Pre- } \\
\text { class preparation and testing; reduction of formal lecture time; team work } \\
\text { during class; focus on class readiness; focus on problem solving skills; } \\
\text { emphasis on student interaction \& engagement; peer-evaluation. } \\
\text { Outcome: Perceived professional growth through motivation; } \\
\text { engagement; critical thinking \& learning effectiveness. Accelerated } \\
\text { development of specialist CCN attributes. }\end{array}$ \\
\hline $\begin{array}{l}\text { Ebadi, } \\
\text { Tabenejad, } \\
\text { Pazokian, } \\
\text { et al, } 2016 \\
53\end{array}$ & $\begin{array}{l}\text { Mixed } \\
\text { methods } \\
\text { validation } \\
\text { study- } \\
\text { Competency } \\
\text { tool }\end{array}$ & $\begin{array}{l}\text { To develop and } \\
\text { test the } \\
\text { psychometric } \\
\text { properties of a } \\
\text { clinical } \\
\text { competence } \\
\text { inventory for } \\
\text { postgraduate } \\
\text { critical care } \\
\text { nursing students. }\end{array}$ & $\begin{array}{l}16 \text { nursing } \\
\text { schools in } \\
\text { Iran }\end{array}$ & $\begin{array}{l}217 \\
\text { postgraduate } \\
\text { critical care } \\
\text { students }\end{array}$ & $\begin{array}{l}\text { Literature review, } \\
60 \text {-items reviewed } \\
\text { for face \& content } \\
\text { validity by a panel of } \\
\text { experts \& students. } \\
\text { Final draft circulated } \\
\text { among } 217 \\
\text { postgraduate } \\
\text { students. } 44 \\
\text { remaining items } \\
\text { examined for } \\
\text { construct validity } \\
\text { using exploratory } \\
\text { factor analysis. } \\
\end{array}$ & MMAT $100 \%$ & $\begin{array}{l}\text { Structure: Competency Inventory for Postgraduate Students of Intensive } \\
\text { Care Nursing: Validity - The final } 44 \text {-item inventory demonstrated a content } \\
\text { validity index of } 0.90 \text {. The content validity ratio ranged from } 0.75-1 \text {. } \\
\text { Reliability - Cronbach's alpha coefficient of } 0.95 \text {, test-retest reliability } 0.96 \\
(p=0.001) \text {. Process: Five included factors comprise: care management; } \\
\text { technical competency; individual management; human-oriented care; } \\
\text { scholarship-oriented care. Outcome: Measurable clinical competence of } \\
\text { postgraduate critical care students }\end{array}$ \\
\hline $\begin{array}{l}\text { Endacott, } \\
\text { Jones, } \\
\text { Bloomer, et } \\
\text { al, } 2015^{54}\end{array}$ & $\begin{array}{l}\text { Descriptive } \\
\text { survey study }\end{array}$ & $\begin{array}{l}\text { To 1) map adult } \\
\text { CCN education } \\
\text { programs, 2) } \\
\text { investigate current } \\
\text { educational } \\
\end{array}$ & $\begin{array}{l}24 \text { European } \\
\text { countries }\end{array}$ & $\begin{array}{l}\text { Descriptive } \\
\text { survey of } \\
\text { registered } \\
\text { nurses in } \\
\text { leadership roles }\end{array}$ & $\begin{array}{l}\text { Survey (telephone, } \\
\text { online \& paper- } \\
\text { based) (non- } \\
\text { validated) }\end{array}$ & $\begin{array}{l}\text { MMAT } 75 \% \\
\text { (Lack of } \\
\text { validated } \\
\text { instrument) }\end{array}$ & $\begin{array}{l}\text { Structure: } 70 \% \text { of European countries provided formal CCN education } \\
\text { programs. } 52 \% \text { of countries recognise CCN as an area of specialty } \\
\text { practice. No Europe-wide recognition or regulation of advanced practice } \\
\mathrm{CCN} \text { roles. Lack of consistency in qualifications awarded. } 54 \% \text { of countries } \\
\text { had no national education standard. } 63 \% \text { of countries had a lack of time }\end{array}$ \\
\hline
\end{tabular}




\begin{tabular}{|c|c|c|c|c|c|c|c|}
\hline & & $\begin{array}{l}\text { structures, } \\
\text { processes \& } \\
\text { outcomes 3) } \\
\text { identify barriers to } \\
\text { progressing CCN } \\
\text { education } \\
\text { programs }\end{array}$ & & $\begin{array}{l}\text { within critical } \\
\text { care } \\
\text { organisations }\end{array}$ & & & $\begin{array}{l}\text { for clinical teaching \& } 45 \% \text { had no protected CCN educator title. } 27 \% \text { had } \\
\text { poor access to educational resources. No consistent standard for } \\
\text { education programs across Europe. Process: Great variability between } \\
\text { program duration ( } 1-24 \text { months) \& eligibility requirements. Lack of } \\
\text { consistency in assessment processes. Outcome: Poor recognition of the } \\
\text { CCN specialty in Europe. Poor fit-for-purpose educational preparation for } \\
\text { increasing critical care patient acuity. Lack of standardisation limits free } \\
\text { movement of CCNs across borders. }\end{array}$ \\
\hline $\begin{array}{l}\text { Gill, Leslie, } \\
\text { Grech et al. } \\
2013^{55}\end{array}$ & $\begin{array}{l}\text { Exploratory } \\
\text { descriptive } \\
\text { qualitative } \\
\text { study }\end{array}$ & $\begin{array}{l}\text { To understand } \\
\text { experiences of } \\
\text { critical care } \\
\text { patients \& families } \\
\text { \& subsequent } \\
\text { opinions on } \\
\text { postgraduate CCN } \\
\text { education \& } \\
\text { outcomes. }\end{array}$ & $\begin{array}{l}\text { Recruitment } \\
\text { from } \\
\text { Australian } \\
\text { health } \\
\text { consumer } \\
\text { organisations }\end{array}$ & $\begin{array}{l}17 \text { participants: } \\
12 \text { divided into } 3 \\
\text { focus groups, } \\
5 \text { provided } \\
\text { individual } \\
\text { interviews }\end{array}$ & $\begin{array}{l}\text { In-depth interviews } \\
\text { audio-recorded. } \\
\text { Thematic analysis }\end{array}$ & MMAT 100\% & $\begin{array}{l}\text { Structure: The CCN role needs specific dimensions of patient \& family- } \\
\text { centred care. There should be minimum standards for CCN course } \\
\text { graduates that encompass patient \& family sensitive processes \& } \\
\text { outcomes. Process: Components of socio-emotional support (talking \& } \\
\text { listening skills; individualising care; relating to stressed people; patient \& } \\
\text { family advocacy) is provided by CCNs inconsistently. Nurse advocacy role } \\
\text { should include arranging family conferences, clarifying medical } \\
\text { information, safe medication administration, facilitating family access to } \\
\text { support, adaptation to the critical care environment, \& facilitating patient } \\
\text { sleep, choice \& dignity. Outcomes: Where physical care \& socio- } \\
\text { emotional support are equally important elements of the CCN role, patients } \\
\text { will feel safe, physical care is provided \& patients \& families will feel } \\
\text { supported. }\end{array}$ \\
\hline $\begin{array}{l}\text { Gill, Leslie, } \\
\text { Grech, et } \\
\text { al. 2014a }{ }^{18}\end{array}$ & $\begin{array}{l}\text { Delphi } \\
\text { validation } \\
\text { study - } \\
\text { Practice } \\
\text { standards }\end{array}$ & $\begin{array}{l}\text { To develop } \\
\text { practice standards } \\
\text { for } \mathrm{CCN} \text { education } \\
\text { in Australia }\end{array}$ & Australia & $\begin{array}{l}\begin{array}{l}\text { Advisory group } \\
(n=25) ; \text { course }\end{array} \\
\text { coordinators } \\
(n=17) ; \text { practice } \\
\text { stakeholders } \\
(n=34) ; \text { \& recent } \\
\text { course } \\
\text { graduates } \\
\text { ( } n=16) .\end{array}$ & $\begin{array}{l}\text { 3-round Delphi study } \\
\text { rating } 84 \text { statements } \\
\text { within } 6 \text { domains } \\
\text { with expert panel of } \\
\text { CCN educators } \\
\text { using } 2 \text { rating scales } \\
\text { (Level of importance } \\
\text { \& level of practice) }\end{array}$ & MMAT 100\% & $\begin{array}{l}\text { Structure: } 98 \text { Practice Standards categorised into } 3 \text { practice outcome } \\
\text { levels with three levels of importance described. } \\
\text { Process: Practice domains include a patient \& family-focused approach; } \\
\text { quality of care \& patient safety; resuscitation; assessment, monitoring \& } \\
\text { data collection; \& critical illness management. Outcome: Graduates of } \\
\text { critical care postgraduate courses should provide independent care for a } \\
\text { variety of ICU patients in most contexts with a patient \& family focus }\end{array}$ \\
\hline $\begin{array}{l}\text { Gill, Leslie, } \\
\text { Grech, et al } \\
2014 b^{19}\end{array}$ & $\begin{array}{l}\text { Mixed } \\
\text { methods } \\
\text { validation } \\
\text { study - } \\
\text { Competency } \\
\text { tool }\end{array}$ & $\begin{array}{l}\text { To develop \& test } \\
\text { a critical care } \\
\text { clinical } \\
\text { assessment tool; } \\
\text { (the SPECT) }\end{array}$ & $\begin{array}{l}\text { Australia } \\
\text { (across } 6 \text { of } 7 \\
\text { states) }\end{array}$ & $\begin{array}{l}\text { Pilot panel (6 } \\
\text { CCNs); Panel } 1 \\
\text { (6 CCNs); Panel } \\
2 \text { (44 CCNs) } \\
\text { from } 4 \\
\text { stakeholder } \\
\text { groups: advisory } \\
\text { group; course } \\
\text { stakeholders; } \\
\text { practice } \\
\text { stakeholders; } \\
\text { course } \\
\text { graduates }\end{array}$ & $\begin{array}{l}\text { Literature review; } \\
\text { pilot survey for face } \\
\text { validity; Expert panel } \\
\text { for content validity; } \\
\text { expert panel for } \\
\text { reliability; 3-round } \\
\text { Delphi study }\end{array}$ & MMAT 100\% & $\begin{array}{l}\text { Structure: The Standard of Practice \& Evaluation of Critical-Care-Nursing } \\
\text { Tool (SPECT) - } 65 \text { standards for course graduates to demonstrate } \\
\text { independently \& } 7 \text { standards for graduates to have knowledge of } / \text { describe. } \\
\text { Process: Domains include patient \& family focussed care; quality of care } \\
\& \text { patient safety; resuscitation; assessment; monitoring \& interpretation; } \\
\text { critical illness management; \& teamwork \& leadership. Outcome: The } \\
\text { SPECT provides a valid \& reliable measure of clinical competence: (mean } \\
\text { content validity index } 0.98 ; \text { intra-rater reliability p }<0.005 ;>\text { moderate } \\
\text { agreement for } 94 \% \text { of responses; Cronbach's alpha }>0.87 \text {. Sufficiently or } \\
\text { very clinically feasible in } 66 \% \text { of responses. }\end{array}$ \\
\hline
\end{tabular}




\begin{tabular}{|c|c|c|c|c|c|c|c|}
\hline $\begin{array}{l}\text { Gill, Leslie, } \\
\text { Grech et al. } \\
2015^{56}\end{array}$ & $\begin{array}{l}\text { Descriptive } \\
\text { qualitative } \\
\text { study }\end{array}$ & $\begin{array}{l}\text { To describe } \\
\text { existing graduate } \\
\text { practice outcomes } \\
\text { for postgraduate } \\
\text { critical care } \\
\text { courses from the } \\
\text { university \& non- } \\
\text { university sector }\end{array}$ & $\begin{array}{l}\text { Australian } \\
\text { adult \& } \\
\text { paediatric } \\
\text { critical care } \\
\text { course } \\
\text { providers } \\
\text { (across all } \\
\text { states) }\end{array}$ & $\begin{array}{l}22 \text { course } \\
\text { providers (18 } \\
\text { university \& } 4 \\
\text { non-university } \\
\text { courses) }\end{array}$ & $\begin{array}{l}\text { Semi-structured } \\
\text { interviews based on } \\
\text { national/international } \\
\text { position statements; } \\
\text { review of course } \\
\text { provider documents } \\
\& \text { websites. } \\
\text { Deductive analytical } \\
\text { interpretation using } \\
\text { Framework } \\
\text { Approach. }\end{array}$ & MMAT $100 \%$ & $\begin{array}{l}\text { Structure: Professional competency standards are the most common } \\
\text { resource for curriculum \& clinical practice assessments. Process: } 21 \text { of } 22 \\
\text { courses included clinical assessments for the Graduate Certificate level } \\
\text { but not for Masters level. Outcome: There is significant variability in } \\
\text { delivery of course content \& expected outcomes. Fifteen courses } \\
\text { prioritised safe practice as a graduate outcome without an expectation for } \\
\text { specialist level practice or team leadership. }\end{array}$ \\
\hline $\begin{array}{l}\text { Gill, } \\
\text { Kendrick, } \\
\text { Davies, et } \\
\text { al. } 201721\end{array}$ & $\begin{array}{l}\text { Mixed } \\
\text { Methods } \\
\text { validation } \\
\text { study- } \\
\text { Practice } \\
\text { standards }\end{array}$ & $\begin{array}{l}\text { To revise the } \\
\text { Australian Practice } \\
\text { Standards for } \\
\text { Critical Care } \\
\text { Nurses (CCNs) }\end{array}$ & $\begin{array}{l}\text { Australian } \\
\text { critical care } \\
\text { education \& } \\
\text { practice } \\
\text { settings } \\
\text { (across all } \\
\text { states) }\end{array}$ & $\begin{array}{l}79 \text { CCNs in } \\
\text { focus groups; } \\
\text { Expert panel of } \\
64 \text { CCNs from } \\
\text { management, } \\
\text { research, clinical } \\
\text { \& education } \\
\text { roles. }\end{array}$ & $\begin{array}{l}\text { Phase 1: } 12 \text { focus } \\
\text { groups } \\
\text { Phase 2: 3-round } \\
\text { modified Delphi } \\
\text { technique. Thematic } \\
\text { analysis. Consumer } \\
\text { feedback provided } \\
\text { on final standards. }\end{array}$ & MMAT $100 \%$ & $\begin{array}{l}\text { Structure: The new competency standards build upon the existing } \\
\text { Nursing \& Midwifery Board of Australia National Competency Standards } \\
\text { for Registered Nurses. The revised structure provides } 15 \text { critical care } \\
\text { practice standards within } 4 \text { domains. Process: Encompasses the domains } \\
\text { of professional practice; provision \& coordination of care; critical thinking \& } \\
\text { analysis; \& collaboration \& leadership. Outcome: Australian standards } \\
\text { provide a guide for curriculum development. }\end{array}$ \\
\hline $\begin{array}{l}\text { Imbracio \& } \\
\text { Sebastiani, } \\
2015^{57} \text {. }\end{array}$ & $\begin{array}{l}\text { Quantitative } \\
\text { postal } \\
\text { survey }\end{array}$ & $\begin{array}{l}\text { To see impact of a } \\
\text { CCN masters on } \\
\text { CCN capacity to } \\
\text { educate, create \& } \\
\text { disseminate new } \\
\text { knowledge, } \\
\text { perform } \\
\text { consultancy } \\
\text { functions \& } \\
\text { demonstrate an } \\
\text { advanced career } \\
\text { path. }\end{array}$ & $\begin{array}{l}4 \text { Italian } \\
\text { Universities }\end{array}$ & $\begin{array}{l}221 \text { previous } \\
\text { Masters in } \\
\text { Critical Care } \\
\text { Nursing students } \\
\text { who studied } \\
\text { between 2003- } \\
2012 \text {. }\end{array}$ & $\begin{array}{l}\text { Online distribution of } \\
\text { a survey }\end{array}$ & $\begin{array}{l}\text { MMAT } 50 \% \\
\text { Response rate } \\
\text { below } 60 \% \\
\text { (34\%). Non- } \\
\text { validated } \\
\text { survey }\end{array}$ & $\begin{array}{l}\text { Structure: Master of Critical care programs are available in Italy. } 93 \% \text { of } \\
\text { Masters graduates did not receive extra remuneration in their roles arising } \\
\text { from their Masters degrees. } 98 \% \text { did not experience vertical career } \\
\text { progression arising from their degree. Process: acquisition of new } \\
\text { knowledge \& skills; increased network of specialist nurse contacts, } \\
\text { exposure to academic settings. Outcomes: a statistically significant } \\
\text { increase in conference presentations ( } 7.2 \%) \text { \& journal publications (5.9\%) } \\
\text { resulted from a Masters education. Frequent workplace recognition of the } \\
\text { specialist role was most evident by junior rather than senior colleagues } \\
(64 \% \text { versus } 24.6 \%) \text {. Advice on technical issues ( } 61.1 \%) \text { \& theoretical } \\
\text { information ( } 58.4 \%) \text { were the most common reasons nurse specialists } \\
\text { were consulted. }\end{array}$ \\
\hline $\begin{array}{l}\text { Lakanmaa, } \\
\text { Suominen, } \\
\text { Perttilä, et } \\
\text { al. } 2014 \\
\text { (34) }\end{array}$ & $\begin{array}{l}\text { Mixed } \\
\text { methods } \\
\text { validation } \\
\text { study- } \\
\text { Competence } \\
\text { measure }\end{array}$ & $\begin{array}{l}\text { To develop \& } \\
\text { validate an } \\
\text { assessment scale } \\
\text { to measure } \\
\text { preliminary } \\
\text { competence (as } \\
\text { opposed to } \\
\text { specialist } \\
\text { competence). }\end{array}$ & $\begin{array}{l}6 \text { university } \\
\text { hospitals \& } 1 \\
\text { polytechnic } \\
\text { hospital in } \\
\text { Finland }\end{array}$ & $\begin{array}{l}\text { Pilot testing: } 18 \\
\text { students \& } 12 \\
\text { nurses. } \\
\text { Psychometric } \\
\text { testing: } 139 \\
\text { students, } 431 \\
\text { nurse }\end{array}$ & $\begin{array}{l}\text { Literature review to } \\
\text { develop scale } \\
\text { content, 2-round } \\
\text { pilot testing \& 2- } \\
\text { round Delphi study }\end{array}$ & $\begin{array}{l}\text { MMAT } 75 \% \\
\text { Psychometric } \\
\text { testing } \\
\text { response rate } \\
\text { below } 60 \% \\
\text { (students } 59 \% \text {, } \\
\text { nurses } 54 \% \text { ) }\end{array}$ & $\begin{array}{l}\text { Structure: The intensive \& critical care nursing competence scale (ICCN- } \\
\text { CS-1) is a } 108 \text {-item self-assessment scale of basic competence. } \\
\text { Process: Measures clinical competence; professional competence; } \\
\text { knowledge base; attitude \& value base } \\
\text { Outcome: High content validity (Delphi consensus of } 80 \%) \& \text { internal } \\
\text { consistency. Satisfactory criterion validity for knowledge base. Cronbach's } \\
\text { alpha } 0.98 \text { for students \& nurses. Useful for self-assessment of basic } \\
\text { competence by graduating nurse undergraduate students \& CCNs. }\end{array}$ \\
\hline $\begin{array}{l}\text { McKinley \& } \\
\text { Elliott, } 2013 \\
58\end{array}$ & $\begin{array}{l}\text { Narrative } \\
\text { Review }\end{array}$ & $\begin{array}{l}\text { To review } \\
\text { evidence of } \\
\text { scholarship among }\end{array}$ & Australia & $\mathrm{N} / \mathrm{A}$ & $\begin{array}{l}\text { Review of Australian } \\
\text { Critical Care Journal } \\
\text { articles from 1988- }\end{array}$ & $\begin{array}{l}\text { Elsevier } \\
\text { Narrative }\end{array}$ & $\begin{array}{l}\text { Structure: Increasing intake of CCNs into Master by research \& doctoral } \\
\text { degrees, increasing number of conjoint, clinical nursing chairs. Australian }\end{array}$ \\
\hline
\end{tabular}




\begin{tabular}{|c|c|c|c|c|c|c|c|}
\hline & & $\begin{array}{l}\text { Australian CCNs } \\
\text { from } 1988-2012 \& \\
\text { to draw links } \\
\text { between } \\
\text { scholarship \& the } \\
\text { progress of CCN } \\
\text { education. }\end{array}$ & & & $\begin{array}{l}2102 \& \text { review of } \\
\text { abstracts from } \\
\text { Australian \& New } \\
\text { Zealand Intensive } \\
\text { Care Society Annual } \\
\text { Scientific Meeting } \\
\text { (1991-2012) } \\
\text { (ANZICS ASM) }\end{array}$ & $\begin{array}{l}\text { review checklist } \\
6 / 7(86 \%) \\
\text { Did not identify } \\
\text { as a narrative } \\
\text { review in the } \\
\text { title. }\end{array}$ & $\begin{array}{l}\text { Critical Care Journal \& ANZICS ASM are a strong platform for critical care } \\
\text { nurse scholarship. } \\
\text { Process: increasing Masters by research \& PhD completions provide an } \\
\text { increasing capacity for research supervision, academic advice \& support. } \\
\text { Sustainable, high-quality dissemination processes (journal publication \& } \\
\text { conference presentation) } \\
\text { Outcomes: Increase in research \& scholarship among CCNs. Increasing } \\
\text { rigour of publications. }\end{array}$ \\
\hline $\begin{array}{l}\text { Williams, } \\
\text { Fullbrook, } \\
\text { Kleinpell, et } \\
\text { al, } 2015^{59}\end{array}$ & $\begin{array}{l}\text { Worldwide } \\
\text { quantitative } \\
\text { survey study }\end{array}$ & $\begin{array}{l}\text { To identify the } \\
\text { activities, concerns } \\
\& \text { expectations of } \\
\text { CCNs \& nursing } \\
\text { organisations } \\
\text { internationally }\end{array}$ & $\begin{array}{l}58 \text { countries } \\
\text { (16 low- } \\
\text { income } \\
\text { countries, } 16 \\
\text { middle- } \\
\text { income } \\
\text { countries, } 27 \\
\text { high-income } \\
\text { countries. }\end{array}$ & $\begin{array}{l}59 \text { respondents } \\
\text { who were } \\
\text { representatives } \\
\text { of national CCN } \\
\text { organisations or } \\
\text { who were } \\
\text { recognised as a } \\
\text { CCN leader in } \\
\text { their country (via } \\
\text { WFCCN } \\
\text { network) }\end{array}$ & $\begin{array}{l}\text { 31-item, 10-point } \\
\text { scale descriptive } \\
\text { questionnaire used } \\
\text { in previous World } \\
\text { Federation of Critical } \\
\text { Care Nurses } \\
\text { (WFCCN) surveys. }\end{array}$ & MMAT $100 \%$ & $\begin{array}{l}\text { Structure: } 73 \% \text { of countries had a national CCN organisation. The } \\
\text { position statements of the European Federation of Critical Care Nursing } \\
\text { Associations (EfCCNa) \& WFCCN have been adopted by several national } \\
\text { organisations. } 9 \text { organisations had published education standards \& } 2 \text { had } \\
\text { adopted EfCCNa standards. Education guidelines available in } 15 \\
\text { countries. Process: } 3 \text { countries were developing education standards. } \\
\text { Specific unmet topics were paediatric conditions; tracheostomy care; } \\
\text { ventilation weaning; sepsis; \& management of pain, sedation \& delirium; } \\
\text { Outcome: Access to CCN education was the highest ranked concern } \\
\text { across countries, with greatest concern from middle \& low-income } \\
\text { countries. Among the range of possible guidelines, guidelines for } \\
\text { education were identified as the greatest need. Development of standards } \\
\text { for CCN education courses were ranked among the most important } \\
\text { activities of CCN organisations, particularly by low \& middle-income } \\
\text { countries. }\end{array}$ \\
\hline
\end{tabular}

Table 2: Characteristics of grey literature (supplementary file)

\begin{tabular}{|l|l|l|l|l|l|l|}
\hline Author & $\begin{array}{l}\text { Document } \\
\text { type }\end{array}$ & Purpose & Jurisdiction & $\begin{array}{l}\text { Key } \\
\text { content }\end{array}$ & $\begin{array}{l}\text { Quality } \\
\text { measure \& } \\
\text { score }\end{array}$ & Recommendations \\
\hline $\begin{array}{l}\text { American } \\
\text { Association } \\
\text { of Critical } \\
\begin{array}{l}\text { Care Nurses } \\
2015^{5}\end{array}\end{array}$ & $\begin{array}{l}\text { Professional } \\
\text { Standard }\end{array}$ & $\begin{array}{l}\text { To define the } \\
\text { scope \& } \\
\text { standards of } \\
\text { critical care } \\
\text { nursing (CCN) } \\
\text { within the } \\
\text { parameters for }\end{array}$ & USA & $\begin{array}{l}\text { Scope of } \\
\text { CCN } \\
\text { practice, } \\
\text { standards } \\
\text { that describe } \\
\text { expected } \\
\text { performance } \\
\text { levels \& }\end{array}$ & $\begin{array}{l}\text { JBI Checklist } \\
\text { for text \& } \\
\text { opinion } 5 / 6 \\
\text { (83\%): } \\
\text { limited } \\
\text { reference to } \\
\text { extant } \\
\text { literature }\end{array}$ & $\begin{array}{l}\text { Structure: The critical care practice environment is led by nurses in interprofessional teams \& this } \\
\text { environment is constantly changing; education providers \& employers should support the } \\
\text { educational advancement \& ongoing learning for CCNs } \\
\text { Process: Specialty practice develops over time; safe quality care requires adequate orientation \& } \\
\text { education. Duration \& content of orientation should be tailored to the individual's needs \& } \\
\text { experience; Standard 3 (Education) requires the nurse to attend ongoing skill training; explore new } \\
\text { sources of knowledge, disseminate knowledge locally \&/or internationally; contribute to the unit's }\end{array}$ \\
\hline
\end{tabular}




\begin{tabular}{|c|c|c|c|c|c|c|}
\hline & & $\begin{array}{l}\text { which the nurse } \\
\text { has been trained. }\end{array}$ & & $\begin{array}{l}\text { addresses } \\
\text { critical care } \\
\text { education. }\end{array}$ & & $\begin{array}{l}\text { education plan; pursue an advanced degree; obtain \& promote specialty certification; \& maintain a } \\
\text { professional portfolio that evidences lifelong learning } \\
\text { Outcomes: Formal \& informal life-long learning enhances competent critical care }\end{array}$ \\
\hline $\begin{array}{l}\text { Critical Care } \\
\text { Networks - } \\
\text { National } \\
\text { Nurse Leads } \\
2015^{23}\end{array}$ & $\begin{array}{l}\text { Quality } \\
\text { assurance } \\
\text { standard (for } \\
\text { post } \\
\text { registration } \\
\text { student } \\
\text { placement) }\end{array}$ & $\begin{array}{l}\text { To define the } \\
\text { responsibilities of } \\
\text { intensive care } \\
\text { units \& education } \\
\text { providers to guide } \\
\text { student } \\
\text { placements } \\
\text { during post } \\
\text { registration critical } \\
\text { care education } \\
\text { programs }\end{array}$ & United Kingdom & $\begin{array}{l}\text { Relationship } \\
\text { with } \\
\text { education } \\
\text { provider } \\
\text { The practice } \\
\text { learning } \\
\text { environment } \\
\text { Workplace } \\
\text { assessment } \\
\text { Student } \\
\text { experience }\end{array}$ & $\begin{array}{l}\text { JBI Checklist } \\
\text { for text \& } \\
\text { opinion } 4 / 6 \\
(67 \%) \text { : } \\
\text { Analytical } \\
\text { process } \\
\text { unclear; } \\
\text { limited } \\
\text { reference to } \\
\text { extant } \\
\text { literature }\end{array}$ & $\begin{array}{l}\text { Structure: Formalised relationship between higher education providers \& critical care practice } \\
\text { environment; honorary contracts for clinical staff; sufficient facilities \& resources in the practice } \\
\text { environment } \\
\text { Process: Regular, structured communication between stakeholders; post course evaluation of } \\
\text { grades, reporting of fails/attrition rates \& practice experience; training for mentors \& assessors; } \\
\text { student rotations where exposure not adequate; competent mentorship; student selection process; } \\
\text { processes for student concerns, professional conduct \& work-health safety issues; assessment } \\
\text { against National Competency Framework; live register of suitably competent \& relevant assessors, } \\
\text { systems for remediation \& failure. Student experience: allocated mentor; defined \& documented } \\
\text { mentor contact hours ( } 40 \% \text { of practice hours), student mentor relationship includes role modelling, } \\
\text { evidence-based care, constructive appraisal \& feedback for staff \& students. Outcome: Programs } \\
\text { are relevant \& meet the needs of students \& industry; the student experience is enhanced. }\end{array}$ \\
\hline $\begin{array}{l}\text { Critical Care } \\
\text { Networks - } \\
\text { National } \\
\text { Nurse Leads, } \\
2015^{4}\end{array}$ & $\begin{array}{l}\text { National } \\
\text { Standard } \\
\text { (Adult Critical } \\
\text { Care Nurse } \\
\text { Education) }\end{array}$ & $\begin{array}{l}\text { To inform } \\
\text { competency } \\
\text { development \& } \\
\text { core curriculum } \\
\text { for the education } \\
\text { of registered } \\
\text { nurses in adult } \\
\text { critical care } \\
\text { settings }\end{array}$ & United Kingdom & $\begin{array}{l}\text { Principles \& } \\
\text { standards for } \\
\text { education } \\
\text { providers \& } \\
\text { critical care } \\
\text { services in } \\
\text { delivery of } \\
\text { post- } \\
\text { registration } \\
\text { critical care } \\
\text { courses }\end{array}$ & $\begin{array}{l}\text { JBI Checklist } \\
\text { for text \& } \\
\text { opinion 6/6 } \\
(100 \%)\end{array}$ & $\begin{array}{l}\text { Structure: National Standards for Adult Critical Care Education; National Competency Framework } \\
\text { (Step } 1 \text { - Foundational transition to practice program; Step } 2 \text { \& } 3 \text { postgraduate critical care } \\
\text { programs of increasing complexity \& decreasing levels of supervision); National core curriculum; } \\
\text { Sponsoring facilities support staff progression \& development \& have a clinical nurse educator for } \\
\text { strategic planning. Mentors demonstrate own ongoing development \& competency. Process: } \\
\text { Curriculum is standardised; covers theory, research, decision-making \& practical skills; is holistic \& } \\
\text { developed with key stakeholders; assessments include theory \& practice, are conducted by } \\
\text { allocated, qualified, experienced assessors \& have strategies to address student failure; courses \& } \\
\text { outcomes evaluated by education providers in consultation with clinical stakeholders; academic } \\
\text { providers are clinically credible \& proficient in theory \& research; students complete a TTP program } \\
\text { prior to postgraduate enrolment, \& have adequate clinical exposure. The learning environment } \\
\text { offers diverse learning experiences, \& minimum of } 40 \% \text { rostered contact between preceptor \& } \\
\text { student; students receive constructive feedback. Outcome: Educational programs are accessible; } \\
\text { responsive; \& adhere to core standards. Validated \& transferrable critical care award. }\end{array}$ \\
\hline $\begin{array}{l}\text { European } \\
\text { Federation of } \\
\text { Critical Care } \\
\text { Nurses } \\
\text { Education } \\
\text { Committee, } \\
2013.60\end{array}$ & $\begin{array}{l}\text { Competency } \\
\text { Framework }\end{array}$ & $\begin{array}{l}\text { To list core } \\
\text { aspects of } \mathrm{CCN} \\
\text { competence to } \\
\text { inform curricula of } \\
\text { postgraduate } \\
\text { CCN education } \\
\text { programs. To } \\
\text { provide a self- } \\
\text { assessment tool } \\
\text { for performance } \\
\text { appraisal for } \\
\text { managers. To } \\
\text { describe } \\
\text { acceptable }\end{array}$ & Europe & $\begin{array}{l}\text { Domains of } \\
\text { CCN practice } \\
\text { where } \\
\text { competency } \\
\text { should be } \\
\text { evident. }\end{array}$ & $\begin{array}{l}\text { JBI Checklist } \\
\text { for text \& } \\
\text { opinion } 4 / 6 \\
(67 \%): \\
\text { Extant } \\
\text { literature not } \\
\text { explicitly } \\
\text { referenced. } \\
\text { Not able to } \\
\text { examine } \\
\text { defence of } \\
\text { incongruence } \\
\text { with literature }\end{array}$ & $\begin{array}{l}\text { Structure: European Federation of Critical Care Nursing Associations } \\
\text { Process: Clinical domains: assessment \& nursing diagnosis; planning; implementation; evaluation. } \\
\text { Professional domains: complex decision-making; ethical \& legal; communication. Management } \\
\text { domain: unit management; team management; maintaining a safe \& supportive environment; } \\
\text { quality assurance. Education \& development domain: personal education \& development; } \\
\text { education \& development of others; evidence-based practice. Outcome: A tool to support } \\
\text { professional \& continuing development of the CCN workforce. }\end{array}$ \\
\hline
\end{tabular}




\begin{tabular}{|c|c|c|c|c|c|c|}
\hline & & $\begin{array}{l}\text { standards of } \\
\text { competence. }\end{array}$ & & & & \\
\hline $\begin{array}{l}\text { Friganovic, } \\
\text { Bloomer, } \\
\text { Northam, et } \\
\text { al } 2016^{61} \text { for } \\
\text { World } \\
\text { Federation of } \\
\text { Critical Care } \\
\text { Nurses. }\end{array}$ & $\begin{array}{l}\text { Position } \\
\text { Statement }\end{array}$ & $\begin{array}{l}\text { To provide } \\
\text { guidance for } \\
\text { culturally sensitive } \\
\text { care for CCNs }\end{array}$ & Worldwide & $\begin{array}{l}\text { Ten universal } \\
\text { principles of } \\
\text { culturally } \\
\text { sensitive } \\
\text { critical care } \\
\text { nursing. }\end{array}$ & $\begin{array}{l}\text { JBI Checklist } \\
\text { for text \& } \\
\text { opinion } 6 / 6 \\
(100 \%)\end{array}$ & $\begin{array}{l}\text { Structure: The Brisbane Declaration on Culturally Sensitive Critical Care Nursing aims to provide } \\
\text { guidance to nurses worldwide. It is supported by the principles of the United Nations' Universal } \\
\text { Declaration of Human Rights \& the International Council of Nurses' Position Statement on Nurses \& } \\
\text { Human Rights. Process: self-assessment; establishing trust; identifying preferred language; } \\
\text { identifying culture, health beliefs \& understanding; ensuring comprehension; culturally appropriate } \\
\text { physical touch; maintenance of modesty \& dignity; impact of gender; consideration of dietary } \\
\text { needs. } \\
\text { Outcome: The } 10 \text { universal principles provide a framework for culturally sensitive critical care to } \\
\text { inform materials \& proarams for CCNs \& CCN education. }\end{array}$ \\
\hline
\end{tabular}




\section{Results}

Twenty-one publications met the inclusion criteria and comprised 13 primary research studies: five were quantitative, ${ }^{18,54,56,57,59}$ two qualitative ${ }^{49,55}$ and six used a mixed-methods design. ${ }^{19,} 21,22,50,52,53$ Five of these were validation studies for practice standards or competency tools. ${ }^{18,19,21,22,53}$ Three used surveys. ${ }^{54,57,59}$ There were two narrative literature reviews ${ }^{51,58}$ and one structured reflection. ${ }^{32}$ Five publications arose from grey literature which included three national or multi-national standards, ${ }^{5,23}$ one competency framework ${ }^{60}$ and one position statement. ${ }^{61}$ Seven publications came from Australia, ${ }^{18,19,21,52,55,56,58}$ four from continental Europe, ${ }^{22,54,57,60}$ three from the UK, ${ }^{4,23,32}$ three from the US ${ }^{5,49,50}$ and one from Iran..$^{53}$ Three explored programs worldwide. ${ }^{51,59,61}$ Quality appraisal using the MMAT, the Narrative Review Checklist and the JBI Checklist for text and opinion papers revealed scores of $100 \%$ of quality items for 11 papers, $4,18,19,21,32,50,53,55,56,59,6175-99 \%$ for six papers ${ }^{5}$, $22,49,52,54,58$ and $50-74 \%$ for four publications. ${ }^{23,51,57,60}$

\section{Thematic description}

Quality CCN education is shaped by, and dependent upon Structure (levels and structures of critical care programs, education frameworks/guidelines and resources for the practice learning environment); Process (processes of the theoretical and practice learning environments, processes for stakeholder engagement and the process of measuring clinical capabilities or competence); and Outcomes (both the scope and the levels of graduate attributes). 
Figure 2. Concept map summarising key review findings

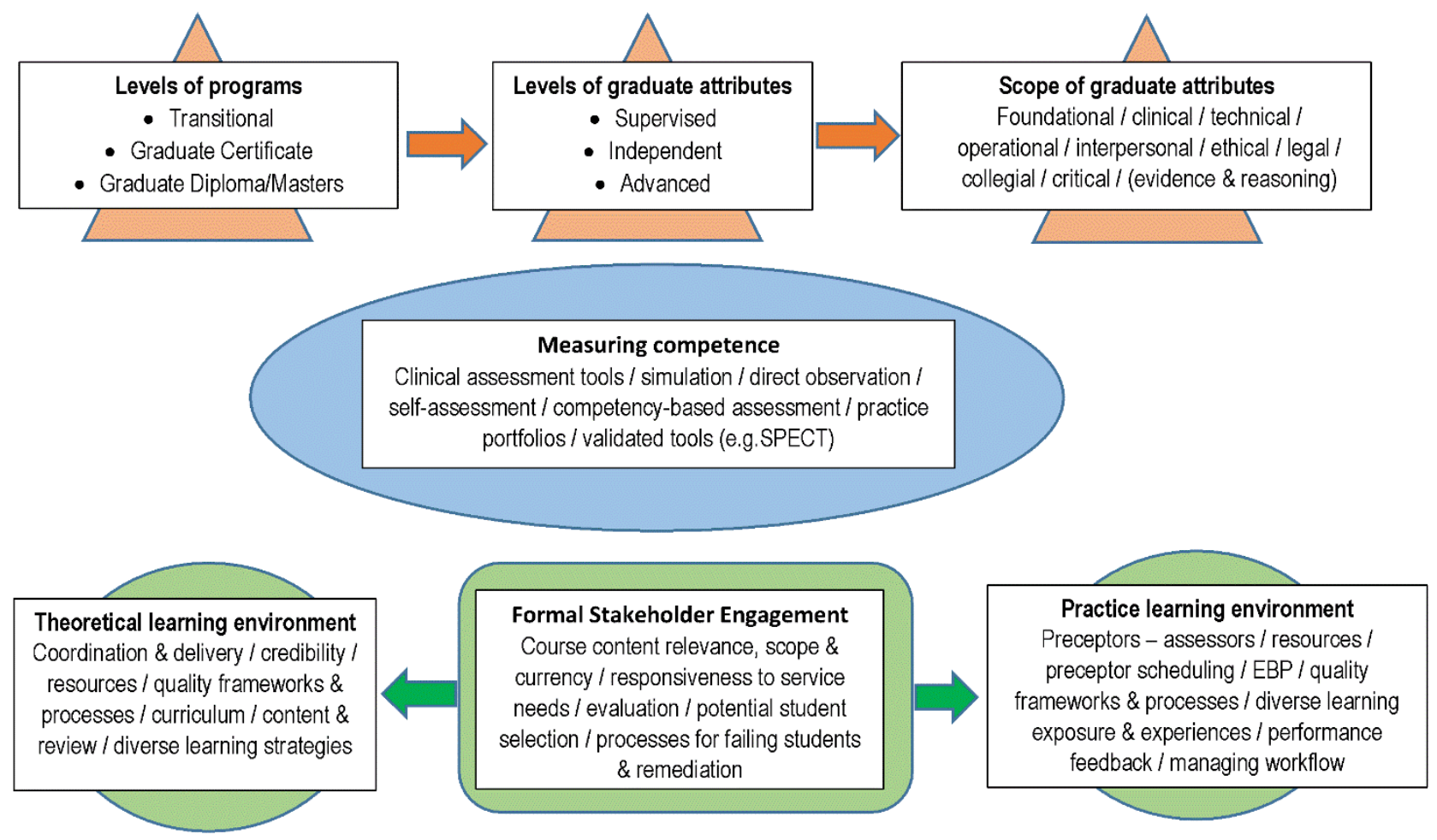

\section{Structure}

Levels and structures of critical care programs

Structure is provided through scaffolded levels of CCN programs for ongoing professional development of the $\mathrm{CCN}$ workforce. These cater for nurses at entry to $\mathrm{CCN}$ practice, through to the development of clinical leaders. At foundational level, in-house hospital-based transition-to-practice (TTP) programs are designed for nurses new to the critical care environment, and are important to the incremental structure of CCN preparation. ${ }^{4}$ These TTP programs, recommended by the UK's Critical Care National Network Nurse Leads Forum (CCN3), are recommended as a precursor to university-provided postgraduate programs. ${ }^{4,32,56}$ TTP programs have a typical duration of 6-12 months, with longer program duration providing time and space to transition, and greater opportunity to examine more complex professional practice issues. ${ }^{49}$ 
Postgraduate courses build on from TTP programs. Postgraduate CCN courses are structured at a range of award levels: Graduate Certificate, Graduate Diploma and Masters, which should be aligned to a higher education standard/framework, and Health Department strategic goals. ${ }^{4}$ In Australia, postgraduate course providers are required to be compliant with the structures of the AQF ${ }^{6}$ and the Higher Education Standards Framework. ${ }^{62}$

In recent literature, most attention is paid to the Graduate Certificate award. It is at Graduate Certificate level that most clinical content is provided, and content usually reflects $\mathrm{CCN}$ education Position Statements. ${ }^{56}$ In the UK, expectations for a Graduate Certificate are for a standard core curriculum matched to learning outcomes, with a prescribed volume of learning over a minimum oneyear period (part-time). While the UK and the US have a standard core CCN curriculum available, no such standard exists in Australia. Minimum entry requirements vary, with around half of Australian Graduate Certificate programs requiring no prior ICU experience and around one-quarter requiring at least 1-year CCN experience. ${ }^{56}$ Graduate Certificate completions are supported in Australia by a continued expectation that a minimum $50 \%$ (and optimum of $75 \%$ ) of $\mathrm{CCNs}$ have a recognised $\mathrm{CCN}$ postgraduate qualification. ${ }^{35}$

There was little attention in the reviewed publications to Graduate Diploma and Masters level courses. A Graduate Diploma is typically 2-years part time with additional content that broadens and deepens knowledge ${ }^{63}$ to support systems thinking and professional leadership. A Masters degree is typically 2.5-3 years part-time, producing autonomous graduates with expert judgement and specialist knowledge and skills for research and/or advanced professional practice. ${ }^{6}$

Variance in best practice is an important international concern. Several publications have revealed the variable quality of available postgraduate CCN courses. For example, the UK's National Standards for Critical Care Nurse Education were recently developed in response to inequities in both cost and perceived value of courses, variations in course quality, and concerns about the perceived endcompetence of bedside nurses. Structurally, in the UK there was variation in the levels of academic 
awards arising from course completion and a lack of transferability of the qualification across geographical boundaries. ${ }^{4}$ In a survey of 24 European countries, similar variation was noted, and was compounded by a lack of national/continental standards for $\mathrm{CCN}$ education, poor resourcing, and an absence of recognition of $\mathrm{CCN}$ practice as a specialty ${ }^{54}$ In Australia, this variation was demonstrated in the types of CCN qualifications awarded, and in the content, assessment and course outcomes ${ }^{56}$

The quality of academic staff is an important structural consideration. Staff facilitating postgraduate programs should be proficient in theory, research and evidence-based practice. They should also be clinically credible, demonstrating relevant practical CCN expertise. ${ }^{4}$

\section{Structures supporting the practice learning environment}

Perhaps the most important structures for the development of safe and effective $\mathrm{CCN}$ practice are the standards and policies that shape the practice learning environment; the subject of several recommendations from the UK Critical Care Nursing Alliance. ${ }^{23}$ This UK document mandates students undertake a minimum of 18 clinical hours per-week in a critical care unit during their program. It also describes minimum resources including a Clinical Nurse Educator, library and information technology access, and adequate number of assessors. The workplace assessment structure is the subject of the UK's Quality Assurance Standards, ${ }^{23}$ while a useful framework for preceptor support is described in the UK Nursing and Midwifery Council's "Standards to Support Learning and Assessing in Practice". ${ }^{24}$ No such national recommendations are in place in Australia, and none were apparent in recent publications arising from other countries.

\section{Processes}

\section{Processes across levels of programs}

Processes incorporate curriculum content and strategies for education delivery and competence assessment. Processes that contribute to TTP program success include an incremental staging of patient acuity and a matching of the theoretical content, aligned with congruent clinical exposure. A 
formal, linear staging checklist to guide participants through clinical topics and skills deemed essential to practice can guide appropriate patient allocation. ${ }^{50}$

While TTP programs have been embraced as a standard approach to CCN preparation, there are aspects of processes that are burdensome and can dilute skill-mix. The goal to pair new nurses with a consistent preceptor (an experienced $\mathrm{CCN}$ allocated as a clinical mentor and/or assessor), while desirable, can make rostering more complex. A lack of preceptor consistency hampers the coordination and continuity of new nurses' professional development and has the potential to overburden experienced CCNs. TTP programs also challenge unit skill mix as the number of transitional nurses makes it difficult to fill vacancies with experienced CCNs. ${ }^{49}$

Much of the literature on processes for postgraduate courses arises from Australia. Educational processes for graduate certificates were examined in a review of 21 Australian postgraduate CCN courses. ${ }^{56}$ Around one-third of Graduate Certificate courses included clinical rotations to different units to maximise experience. Clinical mentorship was embedded into most courses and around threequarters of courses engaged hospital staff as preceptors/assessors within the postgraduate student's own critical care unit. ${ }^{56}$ An identified limitation of Australian Graduate Certificate programs was a lack of a clear vision for course coordination and outcomes, with only a few courses demonstrating a cohesive scaffolding of learning across subjects. ${ }^{56}$ Such scaffolding ensures incremental solidification of clinical understanding and of skills in the appraisal of evidence for practice. It also prevents gaps in essential content.

Internationally, Graduate Diploma and Masters level courses aim to go beyond the specialty clinical content of Graduate Certificates. In Australia, broader professional content is reserved for the Graduate Diploma level, while most Masters' degree components involve a self-directed project and/or a mini-thesis. ${ }^{56}$ Clinical assessments are not included at Masters level. ${ }^{56}$ 
In all CCN learning environments there should be processes to enhance the quality of course coordination and delivery, course credibility, learning and human resources, quality frameworks and processes, curriculum content and review, and diverse learning strategies. In TTP programs, where the theoretical learning environment is the hospital setting, face-to-face time can be challenging to schedule and resource. One descriptive account from the US reported processes for online, learnercentred didactic modules replacing TTP face-to-face study days. ${ }^{50}$

Processes in postgraduate CCN courses should develop capacity for life-long learning where CCN students learn the skills to search, access and critique publications and ideas through reading lists, online discussion boards and critical review of evidence. Students be exposed to multiple evidence sources including scientific, experiential, patient and family accounts, care bundles, guidelines, local practice standards and audits. ${ }^{32}$

Learning also occurs by drawing upon personal experiences and the experiences of others to build a bank of knowledge that informs intuitive practice and underpins adult learning theories. ${ }^{32}$ In the theoretical learning environment, these experiences are shared through in-class and online discussions and reflective exercises. Informed practice is ideally guided by a code of ethics, with students encouraged to consider situations where ethical considerations should be at the forefront of decision-making. ${ }^{32}$

While clinical assessments conducted in real-world situations may be more robust, course providers often use clinical simulation as a valuable complement to theory, and for formative assessment. The fidelity, reliability and validity of such simulations should be considered. ${ }^{32}$ The simulation of nontechnical skills acknowledges the role of human factors in safety and quality, situational awareness and clinical decision-making. ${ }^{32}$

The theoretical learning environment is an ideal place to develop a patient and family focus for care. Strategies include reading lists that incorporate patient and family experiences, and assignments, 
lectures and clinical skills that promote caring and compassion and require consideration of service-

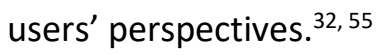

\section{Processes within the practice learning environment}

The processes of the practice learning environment should augment theoretical learning. While it may be challenging logistically, there should be alignment of clinical exposure to the course content and arrangements for alternative clinical placements if exposure is deemed inadequate. A Clinical Nurse Educator should be responsible for coordination of training opportunities and allocation of preceptors. There should be occupational health and safety policies that protect students, along with a process for students to raise concerns about the clinical learning environment. ${ }^{23}$

Clinical preceptors should have knowledge of the clinical capability framework and the course content, with systems to identify practice issues for remediation. ${ }^{23}$ Each clinical unit should keep a database of assessors who can demonstrate their own professional development and clinical currency. Elements that optimise the student experience are a named preceptor for each student, $40 \%$ of the students' practice hours aligned with their preceptor and documented constructive, bi-directional feedback. ${ }^{23}$

\section{Processes for stakeholder engagement}

One of the most important processes of engagement in $\mathrm{CCN}$ education is between the education provider and the clinical setting to create programs that are flexible, accessible and responsive to the needs of industry. ${ }^{4}$ The UK's Quality Assurance Standards ${ }^{23}$ articulate the level of collaboration, the systems and the responsibilities of the workplace learning environment, and their partnership with education providers and students. Important processes include a structured partnership between providers and clinicians to guide planning, evaluation and revalidation of $\mathrm{CCN}$ courses. In an Australian study of graduate CCN education, 14 of 22 courses had some type of collaborative advisory panel or partnership arrangement. ${ }^{56}$ These partnerships were also important for development of robust strategies to address student failure and optimise potential for achievement, to evaluate the practice experience, and to facilitate honorary contracts and training programs for assessors. ${ }^{4,23}$ 


\section{Processes for measurement of competence and capability}

The complexity of measuring competence arises from clinical practice as a complex and multidimensional notion, embodying knowledge, skills, attitudes and values, and shaped by the individual nurse and the context of care. ${ }^{51}$ With $\mathrm{CCN}$ competence being the aim of postgraduate courses, the processes to assess competence should be clear and measurable, with the required level of performance articulated. ${ }^{4}$ National competency frameworks provide the best guidance for competency measurement. ${ }^{4}{ }^{56}$ The most recent, comprehensive discussion of competence for postgraduate CCN program graduates is a literature review of competence among neonatal CCNs. ${ }^{51}$ This review notes that the ambiguity around what competence actually is has contributed to the unsystematic and untested nature of its measures. Measurement of competence should focus on the outcomes of safe practice and effective nurse interventions rather than assessment of tasks or individual skills. ${ }^{4}$

Clinical competence measurement can include both workplace assessment and assessment of written work, all with some limitations. For example, in the measurement processes reviewed by Bromley, ${ }^{51}$ the strategies of direct observation, self-assessment, practice portfolios and clinical assessment tools suffer from poor interrater reliability or have limited research on their overall effectiveness, and may not measure higher-order cognitive skills, attitudes and professionalism. There is however, a validated self-assessment tool now available to measure preliminary $\mathrm{CCN}$ competence for newly-graduating undergraduate students and junior CCNs. ${ }^{22}$

The most common frameworks used to measure competence in Australian Graduate Certificate programs are Benner's ${ }^{64}$ novice-to-expert model and Bondy's ${ }^{65}$ rating scale. ${ }^{56}$ More recently, Australian graduate practice outcomes have been incorporated into a new clinical assessment tool (SPECT) that outlines expected CCN practice attributes. ${ }^{19}$ The SPECT, under psychometric testing, has acceptable levels of clinical feasibility, test-retest reliability, internal consistency, intra-rater reliability, and face and content validity. ${ }^{19}$ If incorporated as minimum criteria and further tested in practice, the 
SPECT provides the opportunity for greater standardisation of Australian CCN course graduate outcomes.

\section{Outcomes}

Scope of graduate attributes

The scope of graduate attributes for postgraduate $\mathrm{CCN}$ courses arise from curriculum programming processes. Through thematic analysis of international documents we have synthesised these attributes into the domains of foundational, clinical, technical, operational, interpersonal, ethical, legal, collegial and critical (see Table 3).

\section{Levels of graduate attributes}

The profession's expectations for postgraduate CCN course graduate attributes (outcomes) depend on the level of the course award. Attributes are commonly discussed using expectations for supervised versus independent versus advanced practice. The UK's “National Standards for Adult Critical Care Nurse Education" ${ }^{4}$ suggests three levels or steps of competency following CCN education. Step 1 covers foundational level competencies that would sit well within a TTP program. TTP program outcomes include improved graduate retention, ${ }^{50}$ reduced patient falls and infection rates, improved pain management and improved patient satisfaction. ${ }^{50}$ In the Australian environment, university education providers commonly acknowledge TTP program completion as credit towards postgraduate CCN courses. While this may result in candidates with greater practical experience, they have a lower level of academic skill. ${ }^{56}$ These Step 1 competencies are ideally built into Step 2 (Graduate Certificate) course entry requirements.

Step 2 activities are outcomes of a CCN postgraduate course (although the level of award is not specified). ${ }^{4}$ Graduate attributes should include a skilled performance of competencies, underpinned by theoretical knowledge and an ability to provide a rationale for practice in relation to policies, procedures and guidelines. More complex problem-solving with a broader variety of patient presentations should be undertaken with minimal supervision. ${ }^{4}$ 
Table 3: Scope of Graduate Attributes

\begin{tabular}{|c|c|}
\hline Attributes & Attribute Description \\
\hline $\begin{array}{l}\text { Foundational } \\
\text { attributes }\end{array}$ & $\begin{array}{l}\text { Foundational attributes encompass safe care delivery to critically ill patients requiring ventilator support, analgaesia, sedation, vasoactive drugs, intravenous fluids \& post-operative care } \\
\text { under direct clinical supervision. A knowledge of anatomy, physiology \& pathophysiology underpins the nurse's subsequent ability to assess, plan, implement \& evaluate care. }{ }^{4} \\
\text { Foundational attributes see an ability to manage disturbances of blood, acid/base, electrolytes \& glucose along with disturbances in the function of body systems. }{ }^{18} \text { Information gathering } \\
\& \text { reporting, patient comfort, time-management \& risk-management are also important expectations for the developing CCN, who should remain aware of their own scope of practice. }{ }^{18,53,} \\
60 \text { Foundational skills are ideally facilitated through TTP programs. } \\
\text { Higher-level clinical attributes should be demonstrated in independent practice following postgraduate qualifications such as a Graduate Certificate. These include applied pharmacology } \\
\& \text { pharmacokinetics, recognition of 'red flags', situational awareness \& a strong level of vigilance \& performance during emergencies \& handover. Management of arrhythmias, cardiac } \\
\text { pacing \& end-of-life care are also commonly cited clinical attributes. }{ }^{4}, 18 \text { More advanced clinical competency sees management of special groups such as cardiac surgery, bariatric \& } \\
\text { obstetric patients, paediatric patients or patients with spinal or burn injury, while advanced practice includes care of patients following interventional cardiology, transplant surgery or } \\
\text { those with a mechanical assist device. } .^{18}\end{array}$ \\
\hline $\begin{array}{l}\text { Technical } \\
\text { attributes }\end{array}$ & $\begin{array}{l}\text { Technical attributes are often required as a foundational skill set \& include care of lines, drains \& endotracheal tubes, invasive \& non-invasive monitoring, aseptic management of wounds } \\
\& \text { physiotherapy techniques. }{ }^{53}\end{array}$ \\
\hline $\begin{array}{l}\text { Operational } \\
\text { attributes }\end{array}$ & $\begin{array}{l}\text { Operational attributes are demonstrated through the management of transfers \& discharge \& by reporting hazards \& incidents. Advanced operational attributes are seen in the } \\
\text { performance of extended roles such as management of admissions \& acting as a resource nurse or access nurse. } .418\end{array}$ \\
\hline $\begin{array}{l}\text { Interpersonal } \\
\text { attributes }\end{array}$ & $\begin{array}{l}\text { Interpersonal attributes include the ability to individualise socio-emotional support \& provide patient \& family education. }{ }^{18} \text { In an Australian qualitative study that described patients \& family } \\
\text { members' experience of intensive or coronary nursing care, the highest value was placed on talking \& listening skills, relating to \& dealing with people under strain, individualising patient } \\
\& \text { family care so that people felt safe, \& patient \& family advocacy. }{ }^{55} \text { In a review of neonatal nurse competency, }{ }^{51} \text { patients \& families also prioritised nurses interpersonal competence \& a } \\
\text { sense of being cared for. A higher level attribute is facilitating family presence during resuscitation. }{ }^{18} \text { Cultural competence is an important interpersonal attribute \& includes the ability to } \\
\text { self-assess one's own cultural positioning, to identify the preferred language \& engage in a range of communication aids, to identify the culture that underpins beliefs, traditions, practices } \\
\text { in health \& illness \& to consider how culture feeds into decision-making \& communication. Cultural competence also considers using physical touch in socially \& culturally appropriate } \\
\text { ways, promoting modesty, considering the impact of gender difference, \& considering food preferences. }{ }^{61} \text { Graduates should also demonstrate the use of closed loop interprofessional } \\
\text { communication in both written \& verbal formats. }{ }^{32}\end{array}$ \\
\hline $\begin{array}{l}\text { Ethical } \\
\text { attributes }\end{array}$ & Ethical attributes are demonstrated through the inclusion of patients \& families in decision-making, \& by maintaining advocacy, privacy, dignity \& confidentiality. 4,18 \\
\hline $\begin{array}{l}\text { Legal } \\
\text { attributes }\end{array}$ & Legal attributes require the nurse to apply their knowledge of legislation, including issues of consent, deprivation of liberty \& mental capacity. ${ }^{4}$ \\
\hline $\begin{array}{l}\text { Collegial } \\
\text { attributes }\end{array}$ & $\begin{array}{l}\text { Collegial attributes demonstrate an ability to establish strengths \& weaknesses of colleagues while providing them with support. A higher level of competence requires the nurse to either } \\
\text { supervise or teach others. } .^{4,18}\end{array}$ \\
\hline $\begin{array}{l}\text { Critical } \\
\text { attributes }\end{array}$ & $\begin{array}{l}\text { Critical attributes are grouped under higher level skills, requiring the nurse to incorporate research evidence into practice \& suggest changes to protocols, policies \& guidelines. }{ }^{18} \text { Critical } \\
\text { competence is also demonstrated through complex problem-solving. }{ }^{4}\end{array}$ \\
\hline
\end{tabular}


While not explicitly stated, it is implied that Step 3 competencies arise from a combination of higherlevel awards (e.g. Graduate Diplomas or Master's degrees) integrated with more extensive clinical experience. At this level, graduate attributes encompass independent practice, the supervision of others, and complex problem-solving where evidence provides the rationale for practice. ${ }^{4}$

The broader professional leadership domain that develops CCN professional practice and the production of new knowledge is notably missing from Step 3 competencies in the UK's National Standards. A Masters' degree in critical care can develop these attributes: a large survey of Italian CCN masters graduates demonstrated increased engagement in conference presentation and research publication. $^{57}$

While the UK's National Standards provide a useful framework, a different approach was taken to understanding and defining postgraduate course practice outcomes in Australia. ${ }^{56} \mathrm{~A}$ multi-phase study identified the expected outcome of a Graduate Certificate graduate as providing safe care to most, but not all critically ill patients, while graduates may require support in complex care or rapidly changing situations. ${ }^{56}$ Graduate outcome standards that define graduate practice capability were divided into activities that should be performed independently, based on their importance to daily practice, with Level 1 having a high level of importance, Level 2 a median rating of importance and Level 3 , the lowest importance. ${ }^{18}$ The most common descriptor of outcome levels was 'competence', with course graduates perceived at the beginning of the specialist expertise continuum. This study found that graduates were not expected to be performing leadership roles such as being a team leader or a resource person; rather, graduates were expected to describe, have knowledge of or perform these roles under supervision. ${ }^{18}$ This expected level of capability is lower than that expressed in European reports and this is thought to be due to Australian nurses increasingly entering postgraduate study earlier in their career. ${ }^{18} \mathrm{CCNs}$ are the youngest cohort across nursing sectors in Australia, ${ }^{66}$ so are earlier in the novice-to-expert ${ }^{64}$ continuum. 
While internationally, most postgraduate $\mathrm{CCN}$ education is provided as content-specific coursework, there are opportunities to engage in higher degree research. The increasing enrolments of Australian CCNs into Masters by research and doctoral degrees has led to important professional outcomes including an increase in scholarship and dissemination of new knowledge by CCN specialists and an increase in critical care academic leadership. ${ }^{58}$

\section{Discussion}

The strength of this review lies in the robust application of an integrative review methodology. ${ }^{44}$ Donabedian's Quality Framework ${ }^{36}$ was useful for reporting the findings of our review and may be useful in future applications to consider the structures, processes and outcomes of interventions or revisions of educational processes informed by these findings. We required a minimum quality score of $50 \%$ for inclusion of articles; over half had a quality score of $100 \%$ and 17 of 21 articles had a quality score of over $75 \%$. It should be noted, however, that while these articles may meet quality requirements within their particular methodological framing, the levels of evidence were frequently low, and this should be considered when applying these results to professional position statements.

A limitation to this review may be the decision to date limit the search to articles published from 2013. We acknowledge the tension between our aim of focussing on contemporary structures processes and outcomes, and the possibility we may have missed valuable insights from earlier work. The dominance of Australian and UK literature in this review reflects a focussed program of research and guideline development in these countries.

Quality education is an outcome of well-articulated and adequately resourced structures and creative, collaborative and evidence-based processes. Of particular importance is the collaborative leadership of professional bodies such as the CCN3 in the UK, the ACCCN in Australia and the ACCN in the US with college members and executives spanning the higher education and health spheres. These 
professional bodies have articulated standards and guidelines to promote a scaffolded approach to CCN education, with the ultimate goal of safe CCN practice.

Perhaps the most useful recent contribution is the articulation of $\mathrm{CCN}$ education as a series of steps. The clarification of TTP programs as a desirable precursor to postgraduate courses is an important addition to CCN education discourse. TTP programs as the foundation of specialist skill development require structural support at a systems level, given the resource-intensive nature of their delivery. ${ }^{67}$ They afford benefits for nurse retention and orientation to the ICU environment, and there is some limited evidence to link these programs to patient outcomes. ${ }^{50}$ A standard TTP curriculum framework, and sharing of resources through, for example, online content within central repositories (e.g. state health department websites) may optimise resource utilisation and overcome equity of access issues, particularly for nurses from rural settings. ${ }^{68}$ Interestingly, a recent Australian report concluded that availability of hospital-based clinical development courses impacts negatively on the uptake of university-based postgraduate programs. ${ }^{10}$ Further examination of this phenomena is worthy of attention. Future research could explore barriers and enablers to uptake of postgraduate programs by individual CCNs.

Higher education providers should not work in isolation from clinical critical care settings. Formal structured and regular engagement between key stakeholders including health consumers ${ }^{33}$ can strengthen curriculum co-design, ${ }^{69,70}$ review and evaluation. Using national standards as a basis for core curriculum ${ }^{4,56}$ can enhance the relevance of postgraduate content and may engender confidence in these programs leading to more CCN graduates. The increasing available body of high quality research, particularly around clinical practice outcome standards for CCN course graduates, may prove a valuable resource for education providers.

The greatest barrier to understanding the issues for postgraduate education is the level of evidence inherent in this literature. While it seems intuitive that well-educated nurses will lead to optimal nursing care, few studies have quantified the impact of postgraduate programs on patient outcomes. ${ }^{10}$ 


\section{Conclusion}

The evidence, standards and guidelines to inform best practice structures and processes for an incremental and ongoing critical care specialist education have been synthesised in this integrative review. Important structures are the stepwise nature or scaffolding of programs, a standard core curriculum, clinically credible staff, national higher education frameworks and guiding education and workforce policies and standards. Facilitating processes are an incremental exposure to patient acuity; consistent and competent hospital-based preceptors/assessors, evidence-based processes for measuring clinical competence; courses delivered with a flexible, modular approach; curricula that incorporates patient and family-centred care and non-technical skills, and stakeholder engagement between the education provider and the clinical setting. Published outcomes included articulation of the scope and levels of graduate attributes for postgraduate $\mathrm{CCN}$ courses and the professional activities associated with each level. There is now a shared professional opportunity to collaborate, implement and evaluate the structures, processes and practices described. There is, however, an acknowledged tension between the expectations of government and professional bodies for policies, standards and position statements to enhance quality and reduce care variance, and the availability of high quality evidence to underpin these across international contexts. For this reason, there should be a renewed focus to design high quality outcomes research to increase the confidence in, and application of, such statements and standards. 


\section{References}

1. Kelly F, Fong K, Hirsch N, Nolan J. Intensive care medicine is 60 years old: the history and future of the intensive care unit. Clinical Medicine. 2014;14:376-9.

2. Jones C. PostTraumatic Stress Disorder - The critical care effect. Australian Critical Care. 2013;26:4-5.

3. Manthous C, Nenbhard I, Hollingshead A. Building effective critical care teams. Critical Care. 2011;15:307.

4. Critical Care Nurses Education Review Forum. National standards for adult critical care education. United Kingdom: Critical Care Networks - National Nurse Leads; 2016.

5. AACN. AACN scope and standards for acute and critical care nursing practice. Aliso Viejo, CA: American Association of Critical Care Nurses; 2015.

6. Australian Qualifications Framework Council. Australian Qualifications Framework. 2011.

7. Ge S, Xi X, Guo G-F. A systematic review of the impact of master's-educated nurses on inpatient care. International Jpournal of Nursing Sciences. 2015;2:414-21.

8. Cotterill-Walker $\mathrm{S}$. Where is the evidence that master's level nursing education makes a difference to patient care? A literature review Nurse Education Today 2012;32:57-64.

9. Twigg D, Myers H, Duffield C, Giles M, Evans G. Is there an economic case for investing in nursing care - what does the literature tell us? . Journal of Advanced Nursing. 2015;71:975-90.

10. Darcy Associates. Review of postgraduate nursing and midwifery education in Victoria. St Kilda2015.

11. Rafferty A, Xyrichis A, Caldwell C. Post-graduate education and career pathways in nursing: a policy brief London: National Nursing Research Unit, King's College; 2015.

12. Haggerty $\mathrm{C}$, Holloway K, Wilson D. Entry to nursing practice preceptor education and support: Could we do it better? . Nursing Praxis in New Zealand. 2012; 28: 30-39;28:30-9.

13. Wang C, Whitehead L, Bayes S. Nursing education in China: Meeting the global demand for quality healthcare. International Journal of Nursing Sciences. 2016;3:131-6.

14. Witt C. Continuing Education: A Personal Responsibility. Advances in Neonatal Care.

2011;11:227-8.

15. Desilets L, Dickerson P, Dickerson P. Continuing nursing education: enhancing professional development. Journal of Continuing Education in Nursing. 2010;41:100-1.

16. Gill F, Lin F, Massey D, Wilson L, Greenwood M, Skylas K, et al. Development of a position statement for Australian critical care nurse education Australian Critical Care. 2018; In Press.

17. Gill F, Leslie G, Grech C, Latour J. A review of critical care nursing staffing, education and practice standards. Australian Critical Care. 2012;25:224-37.

18. Gill F, Leslie G, Grech, C, Boldy D, Latour J. Development of Australian clinical practice outcome standards for graduates of critical care nurse education. Journal of Critical Care Nursing.

2014;24:486-99.

19. Gill F, Leslie G, Grech C, Boldy D, Latour J. Developing and Testing the Standard of Practice and Evaluation of Critical-Care-Nursing Tool (SPECT) for Critical Care Nursing Practice. The Journal of Continuing Education in Nursing. 2014;45:312-23.

20. Williams G, Schmollgruber S, Alberto L. Consensus forum: Worlwide guidelines on the critical care nursing workforce and education standards. Critical Care Clinics. 2006;22:393.

21. Gill F, Kendrick T, Davies H, Greenwood M. A two phase study to revise the Australian Practice Standards for Specialist Critical Care Nurses. Australian Critical care. 2017;30:173-81.

22. Lakanmaa R-L, Suominen T, Perttila J, Ritmala-Castre'n M, Vahlberg T, Leino-Kilpi H. Basic competence in intensive and critical care nursing: development and psychometric testing of a competence scale. Journal of Clinical Nursing. 2014;23:799-810.

23. UK Critical Care Nursing Alliance. Quality assurance standards to underpin student placements in critical care units during post registration critical care educational programmes. Critical Care Network-National Nurse Leads; 2014. 
24. Nursing and Midwifery Council. Standards to Support Learning and Assessing in Practice. London: Nursing \& Midwifery Council; 2008.

25. ACCCN. Position Statement (2006) on the Provision of Critical Care Nursing Education. Australian College of Critical Care Nurses Ltd; 2006.

26. Indian Ministry of Human Resource Development. Architecture of the National Vocational Education Qualifications Framework ( NVEQF). New Delhi2012.

27. QAA. The frameworks for higher education qualifications of UK degree-awarding bodies. Gloucester2016.

28. European Commission. Descriptors defining levels in the European Qualifications Framework (EQF). BrusselsN.D.

29. New Zealand Qualifications Authority. Background to the New Zealand Qualifications Framework. Wellington: New Zealand Government; N.D.

30. Council of Ministers of Education Canada. Ministerial Statement on Quality Assurance of Degree Education in Canada 2007.

31. Bellomo R, Stow P, Hart G. Why is there such a difference in outcome between Australian intensive care units and others? Current Opinion in Anaesthesiology. 2007;20:100-5.

32. Baid H, Hargreaves J. Quality and safety: reflection on the implications for critical care nursing education. British Association of Critical Care Nurses. 2015;20:174-82.

33. Australian Commission on Safety and Quality in Health Care. National Safety and Quality Standards and Accreditation. Safety and Quality Improvement Guide Standard 2: Partnering with Consumers. Sydney: ACSQHC; 2012.

34. CICM. Miniumum standards for intensive care units. College of Intensive care Medicine of Australia and New Zealand; 2011.

35. ACCCN. Workforce Standards for Intensive Care Nursing. Melbourne: Australian College of Critical Care Nurses Ltd.; 2016.

36. Donabedian A. An introduction to quality assurance in health care. 1st ed. New York: Oxford University Press; 2003.

37. Adult CaFEB. Pre-accredited quality framework: Teaching guide. In: Adult CaFE, editor. East Melbourne: State Government of Victoria; 2013.

38. Willett J. Careers Education Quality Framework. Canberra: Enterprise and Career Education Foundation, and the Career Education Association of Victoria; 1999.

39. Ameh S, Gómez-Olivé, Kahn, Tollman S, Klipstein-Grobusch K. Relationships between structure, process and outcome to assess quality of integrated chronic disease management in a rural South African setting: applying a structural equation model. BMC Health Services Research. 2017;17:229. 40. Moore L, VLavoie A, Bourgeois G, Lapointe J. Donabedian's structure-process-outcome quality of care model: Validation in an integrated trauma system. Journal of Acute Care Surgery. 2015;78:116875.

41. Botma Y, Labuschagne M. Application of the Donabedian quality assurance approach in developing an educational programme. Innovations in Education and Teaching International: Taylor \& Francis Online; 2017.

42. Fitzpatrick J. Quality and safety in nursing education. Nursing Education Perspectives. 2014;35:211.

43. McDonald K, Sundaram V, Bravata D. Conceptual Frameworks and Their Application to Evaluating Care Coordination Interventions. Closing the Quality Gap: A Critical Analysis of Quality Improvement Strategies Rockville, MD: Agency for Healthcare Research and Quality (US); 2007.

44. Whittemore R, Knafl K. The integrative review: Updated methodology. Journal of Advanced Nursing. 2005;52:546-53.

45. Moher D, Liberati A, Tetzlaff J, Altman DG ftPG. Preferred reporting items for systematic reviews and meta-analyses: The PRISMA Statement. PLoS MED. 2009;6:e1000097. 
46. Pluye P, Robert E, Cargo M, Bartlett G, O'Cathain A, Griffiths F, et al. Proposal: A mixed methods appraisal tool for systematic mixed studies reviews Montreal, Canada: Department of Family Medicine, McGill University; 2011.

47. Academy of Nutrition \& Dietetics. Narrative Review Checklist. Elsevier; n.d.

48. Joanna Briggs Institute. JBI Critical Appraisal Checklist for narrative, Expert Opinion \& Text. 2014. 49. Adams J, Alexander G, Chisari R, Banister G, McCAuley M, Whitney K, et al. Strengthening New Graduate Nurse Residency Programs in Critical Care: Recommendations From Nurse Residents and Organizational Stakeholders. The Journal of Continuing Education in Nursing. 2015;46:41-8.

50. Bortolotto S. Developing a Comprehensive Critical Care Orientation Program for Graduate Nurses. Journal for Nurses in Professional Development. 2015;31:203-10.

51. Bromley P. Clinical competence of neonatal intensive care nursing students: How do we evaluate the application of knowledge in students of postgraduate certificate in neonatal intensive care nursing? Journal of Neonatal Nursing. 2014;20:140-6.

52. Currey J, Eustace P, Oldland E, Glanville D, Story I. Developing professional attributes in critical care nurses using Team-Based Learning. Nurse Education in Practice. 2015;15:232-8.

53. Ebadi A, Tabanejad Z, Pazokian M, Yasser S. Designing and Psychometric Evaluation of the Competency Inventory for Postgraduate Students of Intensive Care Nursing. Nursing \& Midwifery Studies. 2016;5:e32677.

54. Endacott R, Jones C, Bloomer M, Be Nun M, Iliopoulou K, Egerod I, et al. The state of critical care nursing education in Europe: an international survey. Intensive Care Medicine. 2015;41:2237-40.

55. Gill F, Leslie G, Grech C, Latour J. Health consumers' experiences in Australian critical care units: postgraduate nurse education implications. Nursing in Critical Care. 2013;18:93-102.

56. Gill F, Leslie G, Grech C, Latour J. An analysis of Australian graduate critical care nurse education. Collegian. 2015;22:71-81.

57. Imbracio G, Sebastiani S. Critical care nurse specialist: a descriptive study on the educational implications of Critical Care master's degrees. Scenario. 2015;32:5-18.

58. McKinley S, Elliott D. Twenty-five years of critical care nursing scholarship in Australia. Australian Critical Care. 2013;26:7-11.

59. Williams G, Fulbrook P, Kleinpell R, Schmollgruber S, Alberto L. Critical care nursing organizations and activities: a fourth worldwide review. International Nursing Review. 2015;62:453-61.

60. EfCCNa. EfCCNa Competencies for European Critical Care Nurses. Netherlands: Europena federation of Critical Care Nursing Asssociations; 2013.

61. Friganovic A, Bloomer M, Northam H, Kalauz S, Zellinger M, Lopez V, et al. Brisbane declaration:

Culturally sensitive critical care nursing. World Federation of Critical Care Nurses; 2016.

62. Tertiary Education Quality and Standards Agency. Higher Education Standards Framework

(Threshold Standards). 2011.

63. Universities Australia. Graduate and vocational graduate certificates and diplomas in the AQF.

Canberra: Universities Australia; 2012.

64. Benner P. From novice to expert: Excellence and power in clinical nursing practice. . Menlo Park: Addison Wesley; 1984.

65. Bondy K. Criterion-referenced definitions for rating scales in clinical evaluation. Journal of Nursing Education. 1983;22:376-82.

66. Health Workforce Australia. Australia's future health workforce: Nurses detailed report. Canberra: Commonwealth of Australia; 2014.

67. Morphet J, Considine J, McKenna L. Transition to specialty practice programs in emergency nursing - A review of the literature. Australasian Emergency Journal. 2011;14:45-9.

68. Transition to Intensive Care Steering Group. Transition to Intensive Care Nursing Project: steering group report. 2007.

69. Reid L, Ryan B. Students as co-producers of the curriculum; enhancing student- student engagement? . 6th Annual Learning Innovation Network Conference - Sustainable Models of Student Engagement - Rhetoric or Achievable? Dublin, Ireland2013. 
70. CEDA. Co-design and co-production the future for university curriculums. News and Analysis. Melbourne: Committee for Economic Development of Australia; 2017. 